\title{
On the ionisation fraction in protoplanetary disks
}

\section{Comparing different reaction networks ${ }^{\star}$}

\author{
M. Ilgner and R. P. Nelson
}

Astronomy Unit, Queen Mary, University of London, Mile End Road, London E1 4NS, UK

e-mail: m.ilgner@qmul.ac.uk

Received 21 June 2005 / Accepted 13 September 2005

\begin{abstract}
We calculate the ionisation fraction in protostellar disk models using a number of different chemical reaction networks, including gas-phase and gas-grain reaction schemes. The disk models we consider are conventional $\alpha$-disks, which include viscous heating and radiative cooling. The primary source of ionisation is assumed to be X-ray irradiation from the central star. For most calculations we adopt a specific disk model (with accretion rate $\dot{M}=10^{-7} M_{\odot} \mathrm{yr}^{-1}$ and $\alpha=10^{-2}$ ), and examine the predictions made by the chemical networks concerning the ionisation fraction, magnetic Reynolds number, and spatial extent of magnetically active regions. This is to aid comparison between the different chemical models.

We consider a number of gas-phase chemical networks. The simplest is the five species model proposed by Oppenheimer \& Dalgarno (1974). We construct more complex models by extracting species and reactions from the UMIST data base. In general we find that the simple models predict higher fractional ionisation levels and more extensive active zones than the more complex models. When heavy metal atoms are included the simple models predict that the disk is magnetically active throughout. The complex models predict that extensive regions of the disk remain magnetically uncoupled ("dead") even when the fractional abundance of magnesium $x_{\mathrm{Mg}}=10^{-8}$. This is because of the large number of molecular ions that are formed, which continue to dominate the recombination with free electrons in the presence of magnesium.

The addition of submicron sized grains with a concentration of $x_{\mathrm{gr}}=10^{-12}$ causes the size of the "dead zone" to increase dramatically for all kinetic models considered, as the grains are highly efficient at sweeping up the free electrons. We find that the simple and complex gas-grain reaction schemes agree on the size and structure of the resulting "dead zone", as the grains play a dominant role in determining the ionisation fraction. We examine the effects of depleting the concentration of small grains as a crude means of modeling the growth of grains during planet formation. We find that a depletion factor of $10^{-4}$ causes the gas-grain chemistry to converge to the gas-phase chemistry when heavy metals are absent. When magnesium is included a depletion factor of $10^{-8}$ is required to reproduce the gas-phase ionisation fraction. This suggests that efficient grain growth and settling will be required in protoplanetary disks, before a substantial fraction of the disk mass in the planet forming zone between 1-10 AU becomes magnetically active and turbulent. Only after this has occurred can gas-phase chemical models be used to predict reliably the ionisation degree in protoplanetary disks.
\end{abstract}

Key words. accretion, accretion disks - magnetohydrodynamics (MHD) - solar system: formation - stars: pre-main sequence

\section{Introduction}

Observations of young stars in star forming regions point to the ubiquity of protostellar disks. These disks show evidence for active accretion, with the canonical mass flow rate being $10^{-8} M_{\odot} \mathrm{yr}^{-1}$ (e.g. Sicilia-Aguilar et al. 2004), requiring a means of transporting angular momentum in the disks. At the present time only one mechanism has been shown to work: MHD turbulence generated by the magnetorotational instability (Balbus \& Hawley 1991; Hawley \& Balbus 1991; Hawley et al. 1996).

There are questions, however, about the applicability of the MRI to cool and dense protostellar disks, as the ionisation

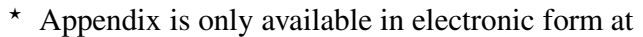
http://wWw.edpsciences.org fraction is expected to be low (e.g. Blaes \& Balbus 1994; Gammie 1996). Magnetohydrodynamic simulations of disks including ohmic resistivity (Fleming et al. 2000) show that for magnetic Reynolds numbers $\mathrm{Re}_{\mathrm{m}}$ smaller than a critical value $\mathrm{Re}_{\mathrm{m}}^{\mathrm{crit}}$, turbulence cannot be sustained and the disks return to a near-laminar state. The value of $\mathrm{Re}_{\mathrm{m}}^{\text {crit }}$ depends on the magnetic field configuration. For net-flux vertical fields turbulence is sustained when $\operatorname{Re}_{\mathrm{m}} \geq 100$. For zero-net flux fields, turbulence dies when $\operatorname{Re}_{\mathrm{m}}<10^{4}$. Although there remains some uncertainty about the precise value of $\mathrm{Re}_{\mathrm{m}}^{\text {crit }}$ due to the possible role of additional non-ideal MHD effects, recent simulations by Sano \& Stone (2002) indicate that the inclusion of Hall E.M.F.s has little impact on the value of $\mathrm{Re}_{\mathrm{m}}^{\mathrm{crit}}$. A value of $\mathrm{Re}_{\mathrm{m}}^{\text {crit }}=100$ typically corresponds to a gas-phase electron fraction of $x\left[\mathrm{e}^{-}\right] \simeq 10^{-12}$ for most disk models. 
The question of whether protostellar disks can sustain MHD turbulence has important implications beyond the issue of how mass accretes onto the central protostar. In particular turbulence may play an important role in planet formation. The growth of planetesimals is controlled by the velocity dispersion (e.g. Wetherill \& Stewart 1993), as is the growth of protoplanetary cores (Kokubo \& Ida 2000; Thommes et al. 2003). Simulations of planets in disks indicate that turbulence may have a significant influence on planet migration and gap formation, as well as the velocity dispersion of planetesimals (Nelson \& Papaloizou 2003, 2004; Winters et al. 2003; Nelson 2005). Obtaining detailed knowledge of the structure of turbulent protoplanetary disks is a crucial part of developing models of planet formation.

A number of studies of the ionisation fraction in protostellar disks have appeared in the literature. The early work by Gammie (1996) put forward the idea that disks may have magnetically "active zones" sustained by thermal or cosmic ray ionisation, adjoining regions that were magnetically inactive "dead zones". More recent studies have examined this issue, but in each case different chemical reaction networks and/or disk models have been employed, making comparison between them difficult. For example, Gammie (1996) used a layered $\alpha$-disk model, and considered cosmic-rays as the primary ionising source. Sano et al. (2000) used the Hayashi (1981) minimum mass solar nebula model in conjunction with a more complex chemical model that included dust grains. Glassgold et al. (1997) and Igea et al. (1999) used the minimum mass model and considered X-rays as the most important ionisation source. Fromang et al. (2002) considered the ionisation fraction in conventional $\alpha$-disk models, and focussed on the role of heavy metals in a simple reaction network. Matsumura \& Pudritz (2003) adopted the externally heated, passive disk model proposed by Chiang \& Goldreich (1997) in conjunction with the Sano et al. (2000) chemical reaction network. Recent work by Semenov et al. (2004) studied chemistry in the D'Alessio et al. (1999) disk model, and employed a complex gas-grain chemical network including most reaction from the UMIST data base.

In this work we study the chemistry and ionisation fraction in conventional $\alpha$-disk models, similar to the approach taken by Fromang et al. (2002). We include X-ray ionisation from the central star as the primary (external) ionisation source, and neglect cosmic-ray ionisation. One of our primary goals is to compare the ionisation fraction predicted by the different reaction networks, within the framework of the same underlying disk model.

We study both gas-phase chemical models, and gas-grain models. We begin by examining the ionisation fraction generated by the simple gas-phase, five species model proposed by Oppenheimer \& Dalgarno (1974) for studying the electron fraction in dark clouds. We study also a number of more complex gas-phase chemical networks. These include the Sano et al. (2000) kinetic model (applied to the gas phase), and networks constructed from various species sets that are drawn from the UMIST data base (Le Teuff et al. 2000). In general we find disagreement between these models, with the more complex networks in particular producing lower fractional ionisation than the simpler schemes. This is because a large number of molecular ions form that are able to recombine quickly with free electrons. Even a relatively large abundance of heavy metals (magnesium) is unable to render the disks fully active in these cases.

We construct a series of gas-grain chemical models that are generalisations of the gas-phase networks. In line with expectations, we find that the addition of submicron sized $\left(r_{\mathrm{gr}}=\right.$ $10^{-5} \mathrm{~cm}$ ) grains causes the magnetically active zone to shrink dramatically, with each of the models being in basic agreement about its size and structure. As a simple approach to modelling the effects of growth during planet formation, we examine the effects of depleting small grains on the chemistry. We find that depletion factors of $10^{-4}$ are required to reproduce the gasphase chemistry for models in which heavy metals are absent. Depletion factors of $\simeq 10^{-8}$, however, are required to reproduce the ionisation fraction obtained when metals are included. The effectiveness of very small numbers of grains in modifying the equilibrium ionisation fraction depends crucially on there being a constant supply of neutral grains, these being generated through recombination reactions between negatively charged grains and positive ions. These models suggest that efficient growth of small grains will be required before they can safely be neglected from chemical networks that predict the ionisation fraction in protoplanetary disks.

This paper is organised as follows. The $\alpha$-disk models we construct are described in Sect. 2. The chemical models are described in Sect. 3, and our scheme for calculating the X-ray ionisation rate is described in Sect. 4. The results of the calculations are presented in Sect. 5, and extensions to the basic models are presented in Sect. 6. Finally we summarise the paper in Sect. 7.

\section{Disk models}

Numerous studies of the ionisation structure in protoplanetary disks have appeared in the literature, with a variety of disk models being used. The resulting ionisation structure differs from model to model, as they differ in their density and temperature profiles. The early study by Gammie (1996) used a layered $\alpha$-disk model. Sano et al. (2000) used the Hayashi (1981) minimum mass solar nebula model, as did Glassgold et al. (1997) and Igea et al. (1999). Matsumura \& Pudritz (2003) adopted the externally heated, passive disk model proposed by Chiang \& Goldreich (1997), and recent work by Semenov et al. (2004) studied chemistry in the D’Alessio et al. (1999) disk model which includes U.V. heating from the central star and internal viscous heating. In this work we study the chemistry and ionisation fraction in conventional $\alpha$-disk models, similar to the approach taken by Fromang et al. (2002).

A detailed description of the disk model and modelling procedure is given in Ilgner et al. (2004). Here we just sketch out the important details. We compute a disk model with inner and outer radii $[0.1,10] \mathrm{AU}$ orbiting with Keplerian velocity about a central star with mass $1 M_{\odot}$. The vertical structure is obtained at a given radius by solving for hydrostatic and thermal equilibrium. Radiative and convective transport is included with opacities taken from Semenov et al. (2003), and disk 


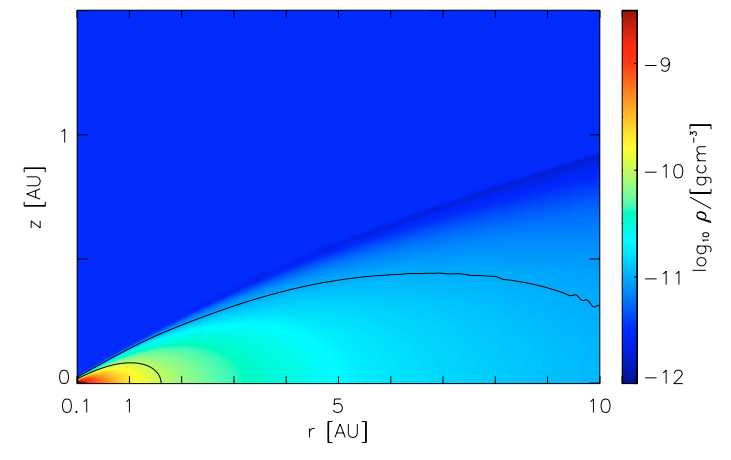

Fig. 1. This contour plot shows the density distribution for our $\alpha=$ $10^{-2}, \dot{M}=10^{-7} M_{\odot} \mathrm{yr}^{-1}$ disk model. The contour lines refer to values of $10^{-11}$ and $10^{-10} \mathrm{~g} \mathrm{~cm}^{-3}$, respectively.

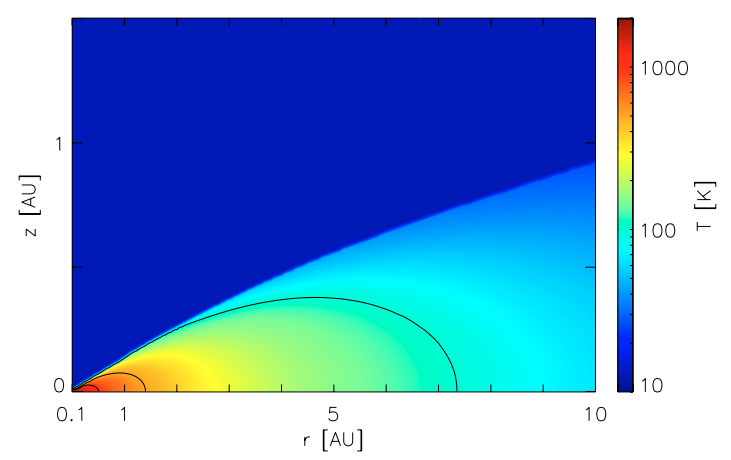

Fig. 2. This contour plot shows the temperature distribution for our $\alpha=10^{-2}, \dot{M}=10^{-7} M_{\odot} \mathrm{yr}^{-1}$ disk model. The contour lines refer to values of 100,500 , and $1000 \mathrm{~K}$, respectively.

heating occurs through viscous dissipation alone. With these ingredients and appropriate boundary conditions the disk model is uniquely specified for given values of $\alpha$ and the mass accretion rate $\dot{M}$. Most of our models are computed with $\alpha=10^{-2}$ and $\dot{M}=10^{-7} M_{\odot} \mathrm{yr}^{-1}$, giving a mass of $0.0087 M_{\odot}$ within the computational domain. Contour plots showing the density and temperature distributions are shown in Figs. 1 and 2. We consider a model with $\alpha=5 \times 10^{-3}$ and $\dot{M}=10^{-8} M_{\odot} \mathrm{yr}^{-1}$ toward the end of this paper. Similar models and procedures for calculating $\alpha$-models were used by Papaloizou \& Terquem (1999) and Fromang et al. (2002).

\section{Chemical models}

We use standard techniques to solve the kinetic equations that describe the chemical models we consider in this paper. We aim to provide sufficient detail to enable an interested reader to reproduce our results, so full descriptions of our procedures are presented in appendices. In this section we begin by describing important aspects of the kinetic models we have implemented, prior to discussing rate coefficients and initial abundances.

\subsection{Kinetic models}

A kinetic model is defined by the components (or species), the chemical reactions that cause time dependent changes in the abundances of species, and by the underlying kinetic equation for each component. The kinetic equations are given by
Table 1. List of reactions (and the corresponding rate coefficients) considered by Oppenheimer \& Dalgarno (1974).

\begin{tabular}{lll}
\hline \hline $1 . \mathrm{m}$ & $\longrightarrow \mathrm{m}^{+}+\mathrm{e}^{-}$ & $\zeta$ \\
$2 . \mathrm{m}^{+}+\mathrm{e}^{-} \longrightarrow \mathrm{m}$ & $\tilde{\alpha}=3 \times 10^{-6} / \sqrt{T} \mathrm{~cm}^{3} \mathrm{~s}^{-1}$ \\
$3 . \mathrm{M}^{+}+\mathrm{e}^{-} \longrightarrow \mathrm{M}+h v$ & $\tilde{\gamma}=3 \times 10^{-11} / \sqrt{T} \mathrm{~cm}^{3} \mathrm{~s}^{-1}$ \\
$4 . \mathrm{m}^{+}+\mathrm{M} \longrightarrow \mathrm{m}+\mathrm{M}^{+} \tilde{\beta}=3 \times 10^{-9} \mathrm{~cm}^{3} \mathrm{~s}^{-1}$ \\
\hline
\end{tabular}

a set of stiff ordinary differential equations which are solved numerically. We have confirmed both algebraically and numerically that conservation of elements and charge is satisfied by our kinetic models. We have implemented the following reaction schemes, and examined the resulting ionisation fractions in $\alpha$-disk models.

\subsubsection{Oppenheimer \& Dalgarno model}

Oppenheimer \& Dalgarno (1974) introduced a simplified reaction scheme to approximate the equilibrium electron abundance in dense interstellar clouds. Using an analytic approximation, this scheme has been used to calculate the electron fraction in protoplanetary disks by a number of authors (Gammie 1996; Glassgold et al. 1997; Fromang et al. 2002).

For a given total particle density $n$, Oppenheimer \& Dalgarno introduced a simple kinetic model involving two elements ("m" and "M"), five components ("m", " $\mathrm{m}^{+}$", "M", " $\mathrm{M}^{+}$", and " $\mathrm{e}^{-}$"), and four chemical reactions. Here, $\mathrm{m}$ and $\mathrm{m}^{+}$ represent a molecule and its ionized counterpart, while $\mathrm{M}$ and $\mathrm{M}^{+}$refer to a heavy neutral metal atom and its ionized counterpart. $\mathrm{e}^{-}$denotes the free electron in the gas phase.

In this model, ions are produced by ionisation and a chargetransfer reaction, while dissociative recombination and radiative recombination are assumed to destroy ions. The reaction set is represented schematically in Table 1 , where $\zeta, \tilde{\alpha}, \tilde{\beta}$, and $\tilde{\gamma}$ are the ionizing flux, the dissociative recombination rate coefficient, the charge-transfer rate coefficient, and the radiative recombination rate coefficient.

One can derive the equilibrium electron abundance analytically using a simple approximation (which is $n \approx n[\mathrm{~m}]$ where $n[\mathrm{~m}]$ denotes the number density of the neutral molecule $\mathrm{m}$ ). The equilibrium electron concentration $x_{\infty}\left[\mathrm{e}^{-}\right]$is given by the roots of the algebraic equation ${ }^{1}$

$x\left[\mathrm{e}^{-}\right]^{3}+\frac{\tilde{\beta} x[\mathrm{M}]}{\tilde{\alpha}} x\left[\mathrm{e}^{-}\right]^{2}-\frac{\zeta}{\tilde{\alpha} n} x\left[\mathrm{e}^{-}\right]-\frac{\tilde{\beta} \zeta x[\mathrm{M}]}{\tilde{\alpha} \tilde{\gamma} n}=0$,

where $x[\mathrm{M}]$ is the equilibrium particle concentration of metals. Neglecting the contributions due to the heavy metal atom Eq. (1) can be reduced to its quadratic form whose solution is

$x_{\infty}\left[\mathrm{e}^{-}\right]=\sqrt{\frac{\zeta}{\tilde{\alpha} n}} \quad$ if $x_{\mathrm{M}}=0$.

In the opposite limit, i.e. assuming $x_{\infty}[\mathrm{M}] \gg x_{\infty}\left[\mathrm{e}^{-}\right]$, similar expressions can be derived depending on further assumption being made. See for example Eq. (27) in Oppenheimer \& Dalgarno (1974) and Eq. (14) in Fromang et al. (2002).

\footnotetext{
${ }^{1}$ For simplicity we dropped the symbol $\infty$ from $x\left[\mathrm{e}^{-}\right]$and $x[\mathrm{M}]$.
} 
Table 2. List of reactions considered by Sano et al. (2000) Notation: $\mathrm{X}^{+}$denotes the ions of the kinetic model: $\left[\mathrm{H}^{+}, \mathrm{H}_{2}^{+}, \mathrm{H}_{3}^{+}, \mathrm{He}^{+}, \mathrm{C}^{+}, \mathrm{m}^{+}\right.$, $\mathrm{Mg}^{+}$] while $\mathrm{Y}, \mathrm{Y}_{\mathrm{ads}}$ refer to the neutral gas-phase species and its adsorbed counterpart onto grain particles, respectively.

\begin{tabular}{|c|c|c|c|c|c|}
\hline $\begin{array}{l}\text { 1. } \mathrm{H}_{2} \\
\text { 2. } \mathrm{H}_{2} \\
\text { 3. } \mathrm{He}\end{array}$ & & $\begin{array}{l}\vec{\longrightarrow} \\
\longrightarrow\end{array}$ & $\begin{array}{l}\mathrm{H}_{2}^{+} \\
\mathrm{H}^{+} \\
\mathrm{He}^{+}\end{array}$ & $\begin{array}{l}+\mathrm{e}^{-} \\
+\mathrm{H} \\
+\mathrm{e}^{-}\end{array}$ & $+\mathrm{e}^{-}$ \\
\hline 4. $\mathrm{H}^{+}$ & $+\mathrm{Mg}$ & $\longrightarrow$ & $\mathrm{Mg}^{+}$ & $+\mathrm{H}$ & \\
\hline 5. $\mathrm{H}^{+}$ & $+\mathrm{O}$ & $\longrightarrow$ & $\mathrm{O}^{+}$ & $+\mathrm{H}$ & \\
\hline 6. $\mathrm{H}^{+}$ & $+\mathrm{O}_{2}$ & $\longrightarrow$ & $\mathrm{O}_{2}^{+}$ & $+\mathrm{H}$ & \\
\hline 7. $\mathrm{H}_{2}^{+}$ & $+\mathrm{H}_{2}^{2}$ & $\longrightarrow$ & $\mathrm{H}_{3}^{+}$ & $+\mathrm{H}$ & \\
\hline 8. $\mathrm{H}_{3}^{+}$ & $+\mathrm{O}^{2}$ & $\longrightarrow$ & $\mathrm{OH}^{+}$ & $+\mathrm{H}_{2}$ & \\
\hline 9. $\mathrm{H}_{3}^{+}$ & $+\mathrm{Mg}$ & $\longrightarrow$ & $\mathrm{Mg}^{+}$ & $+\mathrm{H}_{2}$ & $+\mathrm{H}$ \\
\hline 10. $\mathrm{H}_{3}^{+}$ & $+\mathrm{CO}$ & $\longrightarrow$ & $\mathrm{HCO}^{+}$ & $+\mathrm{H}_{2}^{2}$ & \\
\hline 11. $\mathrm{H}_{3}^{+}$ & $+\mathrm{O}_{2}$ & $\longrightarrow$ & $\mathrm{O}_{2} \mathrm{H}^{+}$ & $+\mathrm{H}_{2}$ & \\
\hline 12. $\mathrm{He}^{+}$ & $+\mathrm{H}_{2}^{2}$ & $\longrightarrow$ & $\mathrm{H}^{+}$ & $+\mathrm{H}^{2}$ & $+\mathrm{He}$ \\
\hline 13. $\mathrm{He}^{+}$ & $+\mathrm{CO}$ & $\longrightarrow$ & $\mathrm{C}^{+}$ & $+\mathrm{O}$ & $+\mathrm{He}$ \\
\hline 14. $\mathrm{He}^{+}$ & $+\mathrm{O}_{2}$ & $\longrightarrow$ & $\mathrm{O}^{+}$ & $+\mathrm{O}$ & $+\mathrm{He}$ \\
\hline 15. $\mathrm{C}^{+}$ & $+\mathrm{Mg}$ & $\longrightarrow$ & $\mathrm{Mg}^{+}$ & $+\mathrm{C}$ & \\
\hline 16. $\mathrm{C}^{+}$ & $+\mathrm{H}_{2}$ & $\longrightarrow$ & $\mathrm{CH}_{2}^{+}$ & $+h v$ & \\
\hline 17. $\mathrm{C}^{+}$ & $+\mathrm{O}_{2}$ & $\longrightarrow$ & $\mathrm{CO}^{+}$ & $+\mathrm{O}$ & \\
\hline 18. $\mathrm{C}^{+}$ & $+\mathrm{O}_{2}$ & $\longrightarrow$ & $\mathrm{O}^{+}$ & $+\mathrm{CO}$ & \\
\hline 19. $\mathrm{HCO}^{+}$ & $+\mathrm{Mg}$ & $\longrightarrow$ & $\mathrm{Mg}^{+}$ & $+\mathrm{HCO}$ & \\
\hline $20 . \mathrm{H}^{+}$ & $+\mathrm{e}^{-}$ & $\longrightarrow$ & $\mathrm{H}$ & $+h v$ & \\
\hline 21. $\mathrm{H}_{3}^{+}$ & $+\mathrm{e}^{-}$ & $\longrightarrow$ & $\mathrm{H}_{2}$ & $+\mathrm{H}$ & \\
\hline 22. $\mathrm{H}_{3}^{+}$ & $+\mathrm{e}^{-}$ & $\longrightarrow$ & $\mathrm{H}^{2}$ & $+\mathrm{H}$ & $+\mathrm{H}$ \\
\hline 23. $\mathrm{He}^{+}$ & $+\mathrm{e}^{-}$ & $\longrightarrow$ & $\mathrm{He}$ & $+h v$ & \\
\hline 24. $C^{+}$ & $+\mathrm{e}^{-}$ & $\longrightarrow$ & $\mathrm{C}$ & $+h v$ & \\
\hline 25. $\mathrm{Mg}^{+}$ & $+\mathrm{e}^{-}$ & $\longrightarrow$ & $\mathrm{Mg}$ & $+h v$ & \\
\hline 26. $\mathrm{HCO}^{+}$ & $+\mathrm{e}^{-}$ & $\longrightarrow$ & $\mathrm{CO}$ & $+\mathrm{H}$ & \\
\hline 27. $X^{+}$ & $+\mathrm{gr}^{2-}$ & $\longrightarrow$ & $\mathrm{gr}^{-}$ & $+\mathrm{Y}$ & \\
\hline 28. $\mathrm{X}^{+}$ & $+\mathrm{gr}^{-}$ & $\longrightarrow$ & $\mathrm{gr}$ & $+\mathrm{Y}$ & \\
\hline 29. $X^{+}$ & $+\mathrm{gr}^{+}$ & $\longrightarrow$ & $\mathrm{gr}^{2+}$ & $+Y_{\text {ads }}$ & \\
\hline 30. $\mathrm{X}^{+}$ & $+\mathrm{gr}$ & $\longrightarrow$ & $\mathrm{gr}^{+}$ & $+Y_{\text {ads }}$ & \\
\hline 31. $\mathrm{e}^{-}$ & $+\mathrm{gr}^{-}$ & $\longrightarrow$ & $\mathrm{gr}^{2-}$ & & \\
\hline 32. $\mathrm{e}^{-}$ & $+\mathrm{gr}$ & $\longrightarrow$ & $\mathrm{gr}^{-}$ & & \\
\hline 33. $\mathrm{e}^{-}$ & $+\mathrm{gr}^{+}$ & $\longrightarrow$ & gr & & \\
\hline 34. $\mathrm{e}^{-}$ & $+\mathrm{gr}^{2+}$ & $\longrightarrow$ & $\mathrm{gr}^{+}$ & & \\
\hline 35. $\mathrm{gr}^{+}$ & $+\mathrm{gr}^{-}$ & $\longrightarrow$ & $\mathrm{gr}$ & $+\mathrm{gr}$ & \\
\hline 36. $\mathrm{gr}^{2+}$ & $+\mathrm{gr}^{2-}$ & $\longrightarrow$ & $\mathrm{gr}$ & $+\mathrm{gr}$ & \\
\hline 37. $\mathrm{gr}^{+}$ & $+\mathrm{gr}^{2-}$ & $\longrightarrow$ & $\mathrm{gr}^{-}$ & $+\mathrm{gr}$ & \\
\hline 38. $\mathrm{gr}^{-}$ & $+\mathrm{gr}^{2+}$ & $\longrightarrow$ & $\mathrm{gr}^{+}$ & $+\mathrm{gr}$ & \\
\hline
\end{tabular}

\subsubsection{Umebayashi \& Nakano model}

Sano et al. (2000) calculated the ionisation fraction in a minimum mass solar nebula disk model adopting a reaction scheme which was investigated originally by Umebayashi \& Nakano (1990) and Nishi et al. (1991) under dense interstellar cloud conditions.

This reaction scheme extends the more schematic gasphase scheme of Oppenheimer \& Dalgarno (1974) by introducing additional neutral and ionized gas-phase species, and gas-grain interactions. Sano et al. (2000) considered 7 ions $\left(\mathrm{H}^{+}, \mathrm{H}_{2}^{+}, \mathrm{H}_{3}^{+}, \mathrm{He}^{+}, \mathrm{C}^{+}, \mathrm{m}^{+}, \mathrm{M}^{+}\right)$and grain particles with seven different grain charges $\left(\mathrm{gr}, \mathrm{gr}^{ \pm}, \mathrm{gr}^{2 \pm}, \mathrm{gr}^{3 \pm}\right)$. The ions $\mathrm{m}^{+}$and $\mathrm{M}^{+}$represent a molecular and metal ion, respectively.

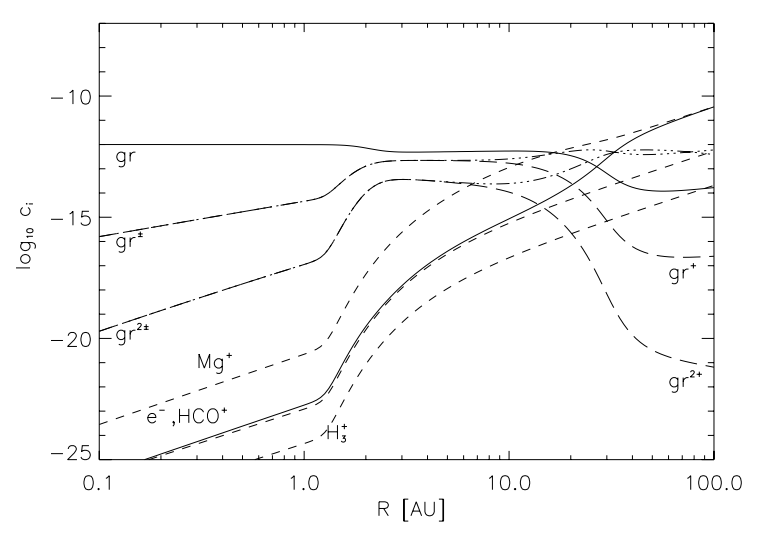

Fig. 3. Equilibrium abundances (per hydrogen nucleus) of some representative particles at the disk midplane, which should be compared to the fiducial model of Sano et al. (2000). The distribution of $\mathrm{e}^{-}$and of $\mathrm{HCO}^{+}$is given by the solid and the dashed line, respectively.

The reactions considered by Sano et al. (2000) are listed in Table 2 which is taken from their paper. Note that the ions $\mathrm{O}^{+}, \mathrm{O}_{2}^{+}, \mathrm{OH}^{+}, \mathrm{O}_{2} \mathrm{H}^{+}, \mathrm{CO}^{+}, \mathrm{CH}_{2}^{+}$, and $\mathrm{HCO}^{+}$are represented by the molecular ion $\mathrm{m}^{+}$(Sano, private communication). Our only omission was triple charged grains, which Sano et al. (2000) included.

The results obtained by Sano et al. (2000) can be used for testing our implementation of this kinetic model. Applying the same physical conditions (which includes a surface density distribution $\Sigma(R)$ based on the minimum mass solar nebula model), the same ionisation flux, and rate coefficients (including the value of $S_{\mathrm{e}^{-}}=0.6$ for all temperatures and grain charges, where $S_{\mathrm{e}^{-}}$denotes the sticking probability of electrons onto neutral or charged grains), and the same equilibrium values of the abundances of neutral components ${ }^{2}$ as Sano et al. (2000) used, we are able to reproduce their results. The equilibrium distributions of the most important components are shown in Fig. 3 and they correspond extremely well to the fiducial model presented in Fig. 1 of Sano et al. (2000). This indicates that our implementation of the Umebayashi \& Nakano reaction scheme is correct.

\subsubsection{Extended kinetic models}

The kinetic models introduced by Oppenheimer \& Dalgarno and by Umebayashi \& Nakano may serve as prototypes for gasphase chemistry and gas-grain chemistry, respectively. Based on these prototypes we have developed more complex kinetic models by extending the number of elements, species, and reactions.

We have formalised our approach to developing extended kinetic models as follows. We start with all gas-phase species listed in Table A.1. This species set contains the elements $\mathrm{H}$, $\mathrm{He}, \mathrm{C}, \mathrm{O}, \mathrm{N}, \mathrm{S}, \mathrm{Si}, \mathrm{Mg}$ and $\mathrm{Fe}$. We extract reactions involving these species from the UMIST database (Le Teuff et al. 2000). The same species set was used by Willacy et al. (1998), Willacy \& Langer (2000), and Markwick et al. (2002) in their studies of

\footnotetext{
${ }^{2}$ We assumed a value of $c_{\text {gr }}=10^{-12}$ for the concentration of grain particles per hydrogen nuclei. $r_{\mathrm{gr}}=0.1 \mu \mathrm{m}$ is taken as the radius of the grain particle.
} 
Table 3. List of generalised reactions determining the mantle chemistry. Neutral gaseous species are represented by the symbol $\mathrm{X}$, its ionised counterpart by $\mathrm{X}^{+}$. The notation $\mathrm{X}(\mathrm{gr})$ and $\mathrm{X}\left(\mathrm{gr}^{-}\right)$refer to neutral gas-phase species adsorbed onto grain particles with no excess charge and with single negative excess charge, respectively.

\begin{tabular}{llllll}
\hline \hline 1. & $\mathrm{X}$ & + & $\mathrm{gr}^{2-}$ & $\longrightarrow$ & $\mathrm{X}\left(\mathrm{gr}^{2-}\right)$ \\
2. & $\mathrm{X}$ & + & $\mathrm{gr}^{-}$ & $\longrightarrow$ & $\mathrm{X}\left(\mathrm{gr}^{-}\right)$ \\
3. & $\mathrm{X}$ & + & $\mathrm{gr}$ & $\longrightarrow$ & $\mathrm{X}(\mathrm{gr})$ \\
4. & $\mathrm{X}$ & + & $\mathrm{gr}^{+}$ & $\longrightarrow$ & $\mathrm{X}\left(\mathrm{gr}^{+}\right)$ \\
5. & $\mathrm{X}$ & + & $\mathrm{gr}^{2+}$ & $\longrightarrow$ & $\mathrm{X}\left(\mathrm{gr}^{2+}\right)$ \\
6. & $\mathrm{X}\left(\mathrm{gr}^{2+} \mathrm{gr}^{ \pm}, \mathrm{gr}^{2 \pm}\right)$ & & & $\longrightarrow$ & $\mathrm{X}$ \\
7. & $\mathrm{X}^{+}$ & + & $\mathrm{gr}^{+}$ & $\longrightarrow$ & $\mathrm{X}\left(\mathrm{gr}^{2+}\right)$ \\
8. & $\mathrm{X}^{+}$ & + & $\mathrm{gr}^{+}$ & $\longrightarrow$ & $\mathrm{X}\left(\mathrm{gr}^{+}\right)$ \\
\hline
\end{tabular}

disk chemistry. This forms the basis of the gas-phase chemical network that we implement.

We also consider chemical evolution due to gas-grain collisions and grain-grain collisions. As yet there is no UMIST-like database that describes these types of reactions, so we have developed our own model. We use a nomenclature that differentiates between reactions that involve mantle species and those that do not. We refer to the former as "mantle chemistry" and the latter as "grain chemistry". We now describe the mantle chemistry, followed by the grain chemistry.

Consider first the neutral components of the previously described gas-phase chemistry. We define a new class of species: mantle species - which are the adsorbed counterparts of the gas-phase neutral species, with the exception of helium which is assumed to remain in the gas phase. These reactions are represented schematically in Table 3, where " $\mathrm{X}$ " is a neutral species and the "gr" are grain particles whose charges are denoted by their indices. A collision between a neutral species $\mathrm{X}$ and a grain with charge $q$ simply results in the formation of a mantle species $\mathrm{X}\left(\mathrm{gr}^{q}\right)$ which physically represents atoms/molecules of species X sitting on grains with charge $q$.

We now consider the "grain chemistry". The reactions are represented schematically in Table 4. Reactions 1 and 2 show that a positive ion colliding with a negative grain results in the ion being neutralised, but remaining in the gas phase, and the grain being left with one less charge - provided a neutral counterpart $\mathrm{X}$ of the ion $\mathrm{X}^{+}$exists in the UMIST database. Collisions between electrons and grains neutralise positive grains, and negatively charge neutral or singlynegative charged grains. The model incorporates single and double charging of grains. Grain-grain collisions result in conservation of grain number but involve charge exchange. There are some species in the set described in Table A.1 whose collisions with grains cannot be described by these reactions. In particular these are collisions between grains and those ions $\mathrm{X}^{+}$(such as $\mathrm{H}_{3}^{+}$and $\mathrm{NH}_{4}^{+}$) with no neutral counterpart. The products of these collisions are unknown. By neglecting these particular collisions between ions and grains one may overestimate the ionisation fraction. To remedy this we have assumed that collisions between ions $\mathrm{X}^{+}$and negatively charged grains
Table 4. List of generalised reactions determining the grain chemistry. $\mathrm{X}^{+}$denotes those components of the species set which have a neutral counterpart X.

\begin{tabular}{rlllllll}
\hline \hline 1. & $\mathrm{X}^{+}$ & + & $\mathrm{gr}^{2-}$ & $\longrightarrow$ & $\mathrm{gr}^{-}$ & + & $\mathrm{X}$ \\
2. & $\mathrm{X}^{+}$ & + & $\mathrm{gr}^{-}$ & $\longrightarrow$ & $\mathrm{gr}^{-}$ & + & $\mathrm{X}$ \\
3. & $\mathrm{e}^{-}$ & + & $\mathrm{gr}^{-}$ & $\longrightarrow$ & $\mathrm{gr}^{2-}$ & & \\
4. & $\mathrm{e}^{-}$ & + & $\mathrm{gr}$ & $\longrightarrow$ & $\mathrm{gr}^{-}$ & & \\
5. & $\mathrm{e}^{-}$ & + & $\mathrm{gr}^{+}$ & $\longrightarrow$ & $\mathrm{gr}^{2}$ & & \\
6. & $\mathrm{e}^{-}$ & + & $\mathrm{gr}^{2+}$ & $\longrightarrow$ & $\mathrm{gr}^{+}$ & & \\
7. & $\mathrm{gr}^{+}$ & + & $\mathrm{gr}^{-}$ & $\longrightarrow$ & $\mathrm{gr}$ & + & $\mathrm{gr}$ \\
8. & $\mathrm{gr}^{2+}$ & + & $\mathrm{gr}^{2-}$ & $\longrightarrow$ & $\mathrm{gr}^{2}$ & + & $\mathrm{gr}$ \\
9. & $\mathrm{gr}^{+}$ & + & $\mathrm{gr}^{2-}$ & $\longrightarrow$ & $\mathrm{gr}^{-}$ & + & $\mathrm{gr}$ \\
10. & $\mathrm{gr}^{-}$ & + & $\mathrm{gr}^{2+}$ & $\longrightarrow$ & $\mathrm{gr}^{+}$ & + & $\mathrm{gr}$ \\
\hline
\end{tabular}

form the same products as the dissociative reactions in the gas phase:

$$
\begin{aligned}
& \mathrm{X}^{+}+\mathrm{gr}^{-} \longrightarrow \mathrm{Y}+\mathrm{Z}+\mathrm{gr} \\
& \mathrm{X}^{+}+\mathrm{e}^{-} \longrightarrow \mathrm{Y}+\mathrm{Z}
\end{aligned}
$$

Quite a few of the dissociative recombination reactions in the UMIST database are branch reactions. We did not use the same branching ratio for the corresponding gas-grain collision. Instead, we assumed that each branch is equally weighted and that the weights are normalised, i.e. $\sum_{i} g_{i}=1$.

We note that gas-grain models have been considered by Willacy et al. (1998), Willacy \& Langer (2000) and Markwick et al. (2002). These authors, however, did not include charged grains in their models. The models computed by Sano et al. (2002) did include charged grains.

We have computed a number of models using gas-phase chemistry only, and gas-phase plus mantle and grain chemistry. We now describe the implementation of these different models.

\subsubsection{Model setup}

The kinetic models we have constructed and applied are listed in Table 5. Note that for the fractional abundance of grains $x_{\mathrm{gr}} \rightarrow 0$, model 4 and model 7 reduce to model 1 and model3, respectively.

We considered three different kinetic models of the gasphase chemistry:

1) model 1: the first and the simplest one is the reaction scheme introduced by Oppenheimer \& Dalgarno (1974). Species M can be represented by any neutral metal atom, and species $m$ represents molecular hydrogen. The model is described in detail in Sect. 3.1.1.

2) mode12: this kinetic model used two elements, similar to the Oppenheimer \& Dalgarno model. All reactions involving hydrogen and magnesium only were extracted from the UMIST database. Note that the reactions involved here differ from the Oppenheimer \& Dalgarno model.

3) mode13: all species listed in Table A.1 were included, giving the most complex kinetic model we have studied so far. The 
Table 5. List of kinetic models. Models with indices 1 and 5 refer to both prototypes discussed in the text. The remaining models refer to the extended models. While models with indices lower than 4 refer to pure gas-phase chemistry only, models with indices 4,6 , and 7 include grain and mantle chemistry.

\begin{tabular}{lcccc}
\hline \hline & \# elements & \# species & \# reactions & grains \\
\hline model1 & 2 & 5 & 4 & \\
model2 & 2 & 8 & 18 & \\
model3 & 9 & 174 & 1966 & \\
\hline model4 & 2 & 12 & 24 & $\sqrt{ }$ \\
mode15 & 5 & 13 & 38 & $\sqrt{ }$ \\
model6 & 5 & 115 & 1090 & $\sqrt{ }$ \\
model7 & 9 & 248 & 2586 & $\sqrt{ }$ \\
\hline
\end{tabular}

model includes the elements: $\mathrm{H}, \mathrm{He}, \mathrm{C}, \mathrm{O}, \mathrm{N}, \mathrm{S}, \mathrm{Si}, \mathrm{Mg}$, and Fe. The same set of gaseous species was adopted by Willacy et al. (1998), Willacy \& Langer (2000) and Markwick et al. (2002) in their studies of disk chemistry.

In addition, we considered four different kinetic models which combine gas-phase chemistry with gas-grain processes:

1) mode14: the simplest one is a modification of Oppenheimer \& Dalgarno's model (model1) obtained by adding the grain and mantle chemistry. The elements are (molecular) hydrogen and magnesium, which need to be specified to define the evaporation temperature from grain surfaces.

2) mode15: this refers to the kinetic model by Umebayashi \& Nakano (1990) as applied by Sano et al. (2000). It is described in subsection 3.1.2.

3) model6: this model was designed to improve the kinetic model of Sano et al. (2000) by including all species listed in Table A. 1 containing the 5 elements ( $\mathrm{H}, \mathrm{He}, \mathrm{C}, \mathrm{O}$, and $\mathrm{Mg}$ ). We extracted 79 gaseous species from Table A.1 which include free electrons. 31 of the 78 gaseous species have an adsorbed neutral counterpart on the grains, so making up the mantle species. Evolving the 79 gaseous species requires the extraction of 799 gas-phase reactions from the UMIST database. Applying our schemes for grain and mantle chemistry (see Tables 3 and 4) 291 additional reactions were introduced.

4) mode17: including all the species listed in Table A.1, we construct the most complex kinetic model of gas-phase, grain, and mantle chemistry we have studied so far. Following the same procedure which was used to set up model6, we have evolved 174 gaseous species (including free electrons) and 69 mantle species. The same species set (gaseous + mantle species) was used by Willacy et al. (1998), Willacy \& Langer (2000) and Markwick et al. (2002). 1965 reactions were extracted from the UMIST database accompanied by 621 additional reactions involving grains.

\subsection{Rate coefficients}

Expressions for the rate coefficients can be obtained from macroscopic considerations. The mean collision rate coefficient is given by $\left\langle\sigma_{\mathrm{c}} \boldsymbol{v}\right\rangle$ where $\sigma_{\mathrm{c}}$ and $\boldsymbol{v}$ denote the cross section and the velocity of the molecules. While the rate coefficients of the gas-phase reactions can be calculated easily using information in the UMIST database (Le Teuff et al. 2000), computation of the rate coefficients for gas-grain processes requires more detailed information. We discuss this further in the following sections, but note here that we assume a grain radius of $r_{\mathrm{gr}}=10^{-5} \mathrm{~cm}$ in all calculation described in this paper.

Compared with previous versions of the UMIST database, its latest version (Le Teuff et al. 2000) accounts for the temperature dependence and specifies the valid temperature range for all reactions. Where explicit information about the temperature range is not available, the rate coefficients have been attributed a temperature range of 10-300 K. Since the parameterisation relies on a mathematical fit to complex data over a restricted temperature range, the validity of the rate coefficients must be checked when used outside their temperature range. We used conservative estimates when calculating the (temperature-dependent) rate coefficients: if the rate coefficient decreases with temperature $T$, we always used the low temperature limit $T_{1}$ as a cut-off value; for lower temperatures the rate coefficient was approximated by $T \equiv T_{1}$. If the rate coefficient increases with temperature, we always used the high temperature limit $T_{2}$ as a cut-off value; for higher temperatures the rate coeffficient was approximated by $T \equiv T_{2}$.

\subsubsection{Collisions between ions/electrons and grains}

The rate coefficients were estimated following the paper by Draine \& Sutin (1987). Compared to the approximation of Umebayashi \& Nakano (1980) which is used widely in astrochemistry, Draine \& Sutin included contributions from the Coulomb potential and the polarization of the grain particles induced by the Coulomb field.

After some algebra a dimensionless reduced rate coefficient $\tilde{J}$ can be extracted from the general expression for the rate coefficient. Depending on the ratio $v$ of the charge $Z e$ of the grain particle to the charge $q$ of the gas-phase particle, which relates to the strength of attractive and repulsive Coulomb interactions, an approximate formula for the reduced rate coefficient can be derived. We applied the equations given by Draine $\&$ Sutin (1987), especially Eq. (3.3) for $v=0$, Eq. (3.4) for $v<0$, and Eq. (3.5) for $v>0$.

When calculating the rate of gas-grain particle interaction, we took account of the fact that not every collision leads to sticking to the grain surface by introducing an average probability $S$ - the "sticking coefficient". We calculated the sticking coefficients - which depend on the energy, the mass, the charge of the incident gas-phase particle, the grain charge and the radius of the grain particle - by applying Eqs. (B5), (B6), (B13) which are given in Nishi et al. (1991). While the electron sticking coefficient $S_{\mathrm{e}^{-}}$depends strongly on the temperature and strength of the Coulomb interaction (see Fig. 4), the sticking coefficients $S_{\mathrm{X}^{+}}$for ions depend only weakly on the temperature. For simplicity, we assumed $S_{\mathrm{X}^{+}}=1$ which is a good approximation. 


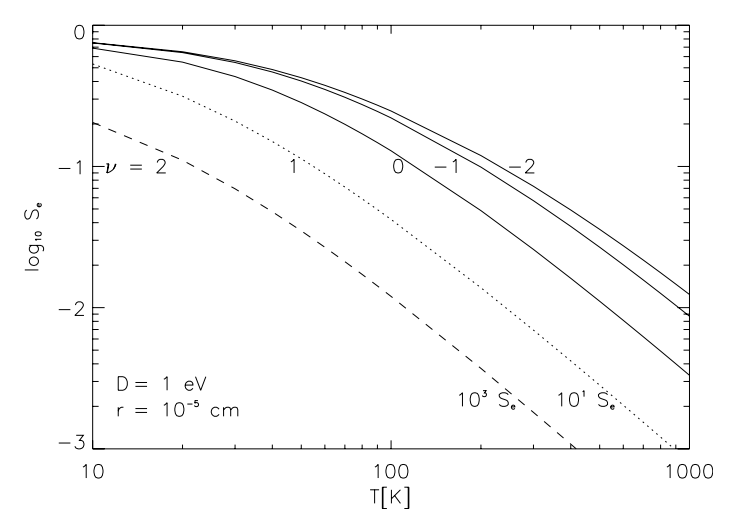

Fig. 4. Sticking probability of an electron onto neutral grains and charged grains as a function of the gas-temperature $T$. Curves are labeled by values of $v=Z e / q$. $D$ and $r$ denotes the polarization potential depth $D$ of the grain and the grain radius, respectively, while $Z e$ and $q$ refer to the grain charge and the charge of the gas-phase particle.

\subsubsection{Collisions between neutral gas-phase particles and grain particles}

We have assumed (see reactions listed in Table 3) that collisions between neutral gas-phase particles and grain particles form mantle species. In order to specify the rate coefficients for these collisions, neutral gas-phase particles are considered to stick onto grain surfaces due to thermal adsorption only. The rate coefficient can be obtained by averaging the kinetic energy $E_{i}$ of the incident gas-phase particles over the thermal energy distribution, assuming that the cross section $\sigma_{\mathrm{c}}$ is independent of $E_{i}$ and is given by the geometrical cross section only. The rate coefficient due to thermal adsorption is then $k=\sigma_{\mathrm{c}}\langle v\rangle$ where $\langle v\rangle$ denotes the mean thermal velocity.

When calculating the sticking rate we accounted for the fact that more than one collision is needed to trap the gas-phase particle onto the grain surface. The procedure for calculating the sticking probability $S_{\mathrm{X}}$ of neutral gas-phase species is similar to that for calculating $S_{\mathrm{e}^{-}}$. The probability $P_{\epsilon}$ that a particle with kinetic energy $E_{i}$ will be adsorbed is approximated by $P_{\epsilon}=\exp \left\{-\epsilon^{2} / 2\right\}$, where $\epsilon$ is given by $\epsilon=E_{i} / \sqrt{D \Delta E_{\mathrm{s}}}$ (see Hollenbach \& Salpeter 1970). $D$ and $\Delta E_{\mathrm{s}}$ denote the dissociation energy and the amount of energy transferred to the grain particle due to lattice vibration, respectively. For each neutral gas-phase component we approximated $D$ by its binding energy for physical adsorption $E_{D}$ (see Table A.2); the value of $\Delta E_{\mathrm{s}}$ was approximated by $2.0 \times 10^{-3} \mathrm{eV}$.

Figure 5 serves as an illustration for the temperature dependency of the sticking probability $S_{\mathrm{X}}$ for different values of dissociation energy.

\subsubsection{Collisions between grain particles}

The rate coefficients for the four different types of grain-grain collisions (see Table 4) were calculated by applying Eq. (3) of Umebayashi \& Nakano (1990). Here the value of the mass density of the grain material $\rho_{\mathrm{gr}}$ is taken to be $\rho_{\mathrm{gr}}=3 \mathrm{~g} \mathrm{~cm}^{-3}$.

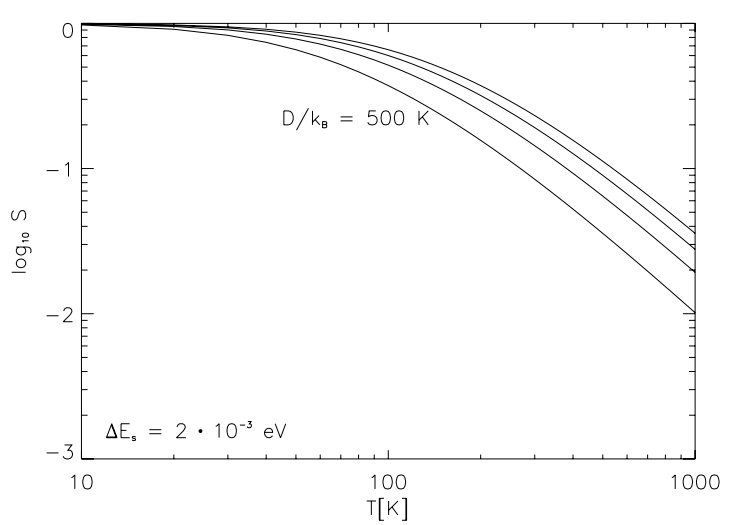

Fig. 5. Sticking probability of neutral species onto grains as a function of the gas-temperature $T . \Delta E_{\mathrm{s}}$ denotes the amount of energy transferred to the grain particle while $D$ is the dissociation energy. The other values of $D / k_{\mathrm{B}}$ are 1000,1500 , and $5000 \mathrm{~K}$.

\subsubsection{Desorption processes}

Desorption processes cause the ejection of mantle species from the grain particles. Here, we considered the thermal desorption of mantle species only. The average time for a mantle species to overcome the surface binding is given by Eq. (2) in Hasegawa et al. (1992), replacing the potential energy barrier between adjacent surface potential energy wells, $E_{\mathrm{b}}$, by the binding energy for physical adsorption $E_{D}$. The binding energy for physical adsorption $E_{D}$ for each considered mantle species is listed in Table A.2. The standard value of $1.5 \times 10^{15} \mathrm{~cm}^{2}$ for the surface density of sites is assumed.

\subsection{Initial abundances}

While the composition of the gas may vary depending on the specific kinetic model, the total particle density $n$ of the gas phase can be considered as a conserved quantity. The variation in the mean molecular weight $\mu$ of the gas due to chemical reactions is negligible as the abundances of molecular hydrogen and helium are approximately constant. Hence the value $\mu=2.33$ is kept constant (assuming a solar composition of the most abundant elements $\mathrm{H}$ and $\mathrm{He}$ ), and the total particle density of the gas phase can be approximated by $n=\rho /\left(\mu m_{\mathrm{H}}\right)$. Here $\rho$ and $m_{\mathrm{H}}$ denote the mass density of the gas and the mass of the hydrogen nuclei, respectively.

We have adopted the same elemental abundances used by previous authors in their studies of the ionisation fraction (Sano et al. 2000; Fromang et al. 2002; Semenov et al. 2004). The abundances of those elements which are not covered by the model of Sano et al. (2000) are taken from Semenov et al. (2004). Hydrogen and helium excepted, all elements are assumed to be depleted in the gas phase compared with solar abundances (shown in Table 6). The fraction $\delta$ of the elements in the gas phase is: 1 for $\mathrm{H}$ and $\mathrm{He}, 0.2$ for $\mathrm{C}$ and $\mathrm{O}, 2.2 \times 10^{-1}$ for $\mathrm{N}, 2.7 \times 10^{-4}$ for $\mathrm{Si}$, and $4.9 \times 10^{-3}$ for $\mathrm{S}$. While the reservoir of all the other elements is fixed by these values, the abundances of metals ( $\mathrm{Mg}$ and $\mathrm{Fe}$ ) are taken as free parameters and will be specified elsewhere. 
Table 6. Elemental abundances per hydrogen nucleus.

\begin{tabular}{lc}
\hline \hline Element & Abundances \\
\hline $\mathrm{H}$ & 1.00 \\
$\mathrm{He}$ & $9.75 \times 10^{-2}$ \\
$\mathrm{C}$ & $3.26 \times 10^{-4}$ \\
$\mathrm{~N}$ & $1.12 \times 10^{-4}$ \\
$\mathrm{O}$ & $8.53 \times 10^{-4}$ \\
$\mathrm{Mg}$ & variable \\
$\mathrm{Si}$ & $3.58 \times 10^{-5}$ \\
$\mathrm{~S}$ & $1.85 \times 10^{-5}$ \\
$\mathrm{Fe}$ & variable \\
$\mathrm{gr}$ & variable \\
\hline
\end{tabular}

The correct choice of initial abundances of chemical species is uncertain as we have no detailed knowledge of how material in molecular clouds evolves as it collapses to form a protostellar disk, or how it evolves during the early stages of disk formation and evolution. We used the simplest approach for tackling this issue. With exception of hydrogen (which is completely locked in molecular form), the elements are taken to be atomic. The number of hydrogen nuclei $n_{\mathrm{H}}$ (which is needed to get the species' particle concentrations) is approximated by $n_{\mathrm{H}}=n / \sum_{i} c_{i}$, where $c_{i}$ refers to the elemental abundances (per hydrogen nucleus) of element $i$ in the gas phase. Note the difference in the notation: $x\left[\mathrm{X}_{i}\right]$ denotes the particle concentration of species $\mathrm{X}_{i}$ normalised to the total particle density $n . x_{i}$ denotes the elemental particle concentration of the element $i$.

Since we consider grain particles with different electric charge excess, only neutral grains were assumed to exist initially, i.e. the total number density $n_{\mathrm{gr}}$ of grain particles is given by $n_{\mathrm{gr}} \equiv n[\mathrm{gr}]$ with $n\left[\mathrm{gr}^{ \pm}\right]=n\left[\mathrm{gr}^{2 \pm}\right]=0$.

\section{Sources of free electrons}

In this work we treat the free electrons $\mathrm{e}^{-}$as an independent component of the reaction network and solve a kinetic equation for their abundance $x\left[\mathrm{e}^{-}\right]$.

Many elementary ionisation processes are considered in the UMIST database. Reactions which may cause the formation of free electrons are: i) photoreactions ii) reactions due to cosmic-ray particles which includes photoreactions induced by cosmic-ray particles and iii) chemiionisation reactions.

We have neglected the effects of the stellar and interstellar ultraviolet radiation field, which drive the photoreactions. There is great uncertainty about the amount of unattenuated UV flux that reaches the disk surface. In addition the exponential decay of this field with increasing column density means that it can only act as source of free electrons in a thin skin at the disk surface. As we are primarily interested in calculating the depth of the magnetically active zones in the disk models, this thin surface layer is unimportant.

Galactic cosmic-ray particles are unlikely to reach the surface of the inner regions of a protostellar disk due to their exclusion by $\mathrm{T}$ Tauri winds. Indeed the solar wind blocks cosmic-ray particles with energies less than $100 \mathrm{MeV}$ from entering the inner solar system. Preferring a conservative estimate of the electron abundance, we did not consider any of the 11 cosmicray particle reactions listed in the UMIST database. For the same reason, we did not consider any of the cosmic-rayinduced photoreactions.

Only one chemiionisation reaction, i.e.

$\mathrm{CH}+\mathrm{O} \longrightarrow \mathrm{HCO}^{+}+\mathrm{e}^{-}$

is tabulated in the UMIST database; the importance of this particular type of (associative) ionisation for the formation of carbon monoxide in interstellar clouds was pointed out by Dalgarno et al. (1973).

Other additional sources of free electrons such as the contributions due X-ray photons, thermal ionisation, and the decay of radioactive elements are analysed in the subsequent Sects. 4.2, 5.1 and 6.0.

\subsection{X-rays}

We have included the effects of X-ray radiation on the chemistry. Since the first X-ray observatories were launched, the $\mathrm{X}$ ray properties of YSOs have been well documented (see the review by Glassgold et al. 2000).

We adopted values $L_{\mathrm{X}}=10^{30} \mathrm{erg} \mathrm{s}^{-1}$ and $k_{\mathrm{B}} T_{\mathrm{X}}=3 \mathrm{keV}$ for the total X-ray luminosity $L_{\mathrm{X}}$ and the plasma temperature $T_{\mathrm{X}}$, respectively, corresponding to a young star of approximately solar mass. Adopting the scheme described by Fromang et al. (2002), we modelled the X-ray source as a coronal ring of radius $10 R_{\odot}$ centered on the star.

\subsection{X-ray ionisation rate}

We assumed that photoelectric absorption of X-ray photons dominates while neglecting Compton scattering. For the X-ray photon energies considered, this assumption simplifies the description of the interaction of X-ray photons with matter in a way that depends on the attenuating column densities. In addition, the results presented by Igea \& Glassgold (1999) indicate that the difference in the total X-ray ionisation rates between the two cases "are about the same at small and large values of the vertical column density and differ by about one order of magnitude at the scattering shoulder".

Following Krolik \& Kallman (1983), we modelled the X-ray flux of the optically thin bremsstrahlung spectrum by the exponential law $F_{\epsilon}=F_{0} \exp \left\{-\epsilon / k_{\mathrm{B}} T_{\mathrm{X}}\right\}$. For larger X-ray optical depths ${ }^{3} \tau_{\mathrm{X}}$, the local X-ray flux $F$ per unit energy is attenuated by $F=F_{\epsilon} \exp \left\{-\tau_{\mathrm{X}}\right\}$. We further assumed that the $\mathrm{X}$-ray ionisation only depends on the elemental composition of the gas and is independent of the species composition. Hence, the absorption of X-ray photons can be simplified by introducing an effective photoionisation cross section (per hydrogen nucleus) $\sigma_{\text {eff }}=\sum_{i} x_{i} \sigma_{i}$. Here $\sigma_{i}$ refers to the partial photoionisation cross section of each atomic species and $x_{i}$ denotes the

3 The X-ray optical depth is given by $\tau_{\mathrm{X}}=\sigma(E) N . \sigma$ is the total gas X-ray photoabsorption cross section per hydrogen nucleus while $N$ denotes the (particle) column density along the line of sight toward the X-ray source. 


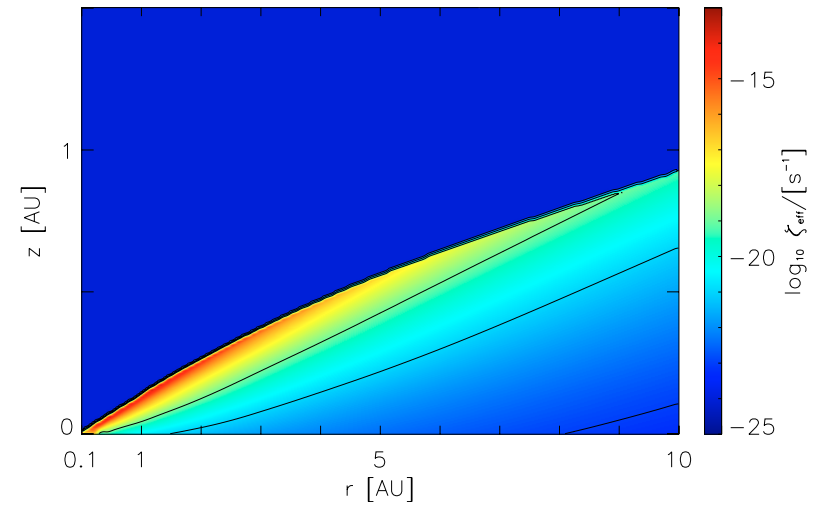

Fig. 6. The effective $X$-ray ionisation rate $\zeta_{\text {eff }}$ per hydrogen nucleus (including contributions due to thermal ionisation of potassium). The disk parameters are $\alpha=10^{-2}$ and $\dot{M}=10^{-7} M_{\odot} \mathrm{yr}^{-1}$. The contour lines refer to values of $\zeta_{\text {eff }}: 10^{-19}, 10^{-21}$, and $10^{-23} \mathrm{~s}^{-1}$.

particle concentration of the element $i$. We used the expression for the effective (total) photoionisation cross section given by Igea \& Glassgold (1999) for the gas-phase depletion of the heavy elements compared to solar abundances.

We calculated the effective $\mathrm{X}$-ray ionisation rate $\zeta_{\text {eff }}$ for hydrogen by assuming that $\zeta_{\text {eff }}$ is dominated by secondary ionisation. The number of secondary ionisations $N_{\mathrm{sec}}$ per unit of primary photoelectron energy is considered to be independent of energy. Finally, the effective X-ray ionisation rate (per hydrogen nucleus) $\zeta_{\text {eff }}$ is approximated by

$\zeta_{\mathrm{eff}} \approx N_{\mathrm{sec}} \int_{E_{\min }}^{\infty} F(E) \sigma_{\mathrm{eff}} \mathrm{d} E$.

The value of $N_{\text {sec }}$ is about 26 for hydrogen if the energy $E$ is measured in units of $\mathrm{keV} . E_{\min }$ denotes the minimum X-ray photon energy. Hence, for values of photon energies less then $E_{\text {min }}$ the photon will be absorbed before the disk surface is reached. Our choice of $E_{\min }=0.1 \mathrm{keV}$ - which is above the required energy for ionisation of $\mathrm{H}_{2}(15.4 \mathrm{eV})$ - accounts for absorption in the source (see Shang et al. 2002).

The integral can be transformed into a dimensionless integral given for example by Eq. (4) in Fromang et al. (2002). By applying the method of steepest descent, this dimensionless integral can be approximated by an analytic asymptotic formula. However, we evaluated the integral numerically and checked it against the value obtained by the asymptotic approximation ${ }^{4}$.

We calculated the X-ray optical depth $\tau_{\mathrm{X}}$ along the line of sight between the X-ray source and the point in question (as did Fromang et al. 2002) ${ }^{5}$.

For a given effective $\mathrm{X}$-ray ionisation rate $\zeta_{\text {eff }}$ we must specify the fraction of $\mathrm{H}^{+}$and $\mathrm{H}_{2}^{+}$ions produced due to $\mathrm{X}$-ray ionisation of $\mathrm{H}_{2}$. We adopted the values given by Cravens \& Dalgarno (1978) taken from calculations of the efficiencies that cosmic rays with energy of $1 \mathrm{MeV}$, traversing a neutral gas of molecular hydrogen, produce these particular ions (see

\footnotetext{
${ }^{4}$ Note the typo in Eq. (5) of Fromang et al. (2002), with $b$ instead of $-b$.

5 Though this particular matter is not explicitly described in Semenov et al. (2004), the tabulated data indicate they calculated $\tau_{\mathrm{X}}$ along the line of sight too.
}

Table 7.

\begin{tabular}{llllllll}
\hline \hline 1. & $\mathrm{H}_{2}$ & $\longrightarrow$ & $\mathrm{H}_{2}^{+}$ & + & $\mathrm{e}^{-}$ & & \\
2. & $\mathrm{H}_{2}$ & $\longrightarrow$ & $\mathrm{H}^{+}$ & + & $\mathrm{H}$ \\
3. & $\mathrm{He}$ & $\rightarrow$ & $\mathrm{He}^{+}+\mathrm{e}^{-}$ & $0.03 \cdot \zeta_{\text {eff }}$ \\
& + & $\mathrm{e}^{-}$ & & $0.84 \cdot \zeta_{\text {eff }}$ \\
\hline
\end{tabular}

Table 7). Contributions from secondary ionisation were included too. Cravens \& Dalgarno found that at proton energies of $1 \mathrm{MeV}$ for example, the total ionisation rate (which is the sum of primary and secondary rates) is larger than the primary rate by a factor of 1.44 .

Since our kinetic models do not include photoreactions driven by incident FUV flux, we have simplified the terminology used in certain sections of this paper. Instead of "effective X-ray ionisation rate" we often use the phrase "photoionisation".

\section{Results}

The majority of our calculations used an $\alpha$-disk model with $\alpha=10^{-2}$ and $\dot{M}=10^{-7} M_{\odot} \mathrm{yr}^{-1}$. We have evolved the disk chemistry using the kinetic models described in Sect. 3, with the primary purpose of examining the size of the "active zone" (the meaning of this phrase is explained below). One primary aim of this work is to compare and understand the differences in the size of the "active" zones obtained with each of these chemical networks, under physical conditions that are otherwise the same. While a number of studies of the ionisation fraction in protostellar disk models have appeared in the literature, they have each used different kinetic and/or disk models, so that direct comparison between them has not been possible.

As outlined in the introduction, the electron fraction is of great importance in protoplanetary disks as it determines how well the gas is coupled to the magnetic field, and hence whether MHD turbulence can be sustained by the MRI. The important discriminant is the magnetic Reynolds number $\mathrm{Re}_{\mathrm{m}}$, which we define as

$\operatorname{Re}_{\mathrm{m}}=\frac{H c_{\mathrm{s}}}{\mu_{\mathrm{m}}}$

where $H$ is the disk semi-thickness, $c_{\mathrm{s}}$ is the sound speed, and $\mu_{\mathrm{m}}$ is the magnetic diffusivity (not to be confused with the mean molecular weight). Numerical simulations (e.g. Fleming et al. 2000) indicate that a critical value of the magnetic Reynolds number, $\mathrm{Re}_{\mathrm{m}}^{\text {crit }}$, exists such that non linear MHD turbulence cannot be sustained if $\operatorname{Re}_{\mathrm{m}}$ falls below $\mathrm{Re}_{\mathrm{m}}^{\text {crit }}$. The value of $\operatorname{Re}_{\mathrm{m}}^{\text {crit }}$ depends on the field geometry. For a net flux vertical field $\mathrm{Re}_{\mathrm{m}}^{\text {crit }} \simeq 10^{2}$, and for a zero net flux field $\mathrm{Re}_{\mathrm{m}}^{\text {crit }} \simeq 10^{4}$. A recent numerical study of MRI driven turbulence including the Hall effect indicates that $\mathrm{Re}_{\mathrm{m}}^{\mathrm{crit}}$ is not particularly sensitive to this effect (Sano \& Stone 2002). The magnetic diffusivity $\mu_{\mathrm{m}}$ expressed in c.g.s. units is (e.g. Blaes \& Balbus 1994)

$\mu_{\mathrm{m}}=\frac{234}{x\left[\mathrm{e}^{-}\right]} T^{1 / 2}$

Given the electron fraction $x\left[\mathrm{e}^{-}\right]$from the kinetic models, and physical conditions within the disk, we are thus able to calculate the spatial variation of $\mathrm{Re}_{\mathrm{m}}$ inside the disk models. 


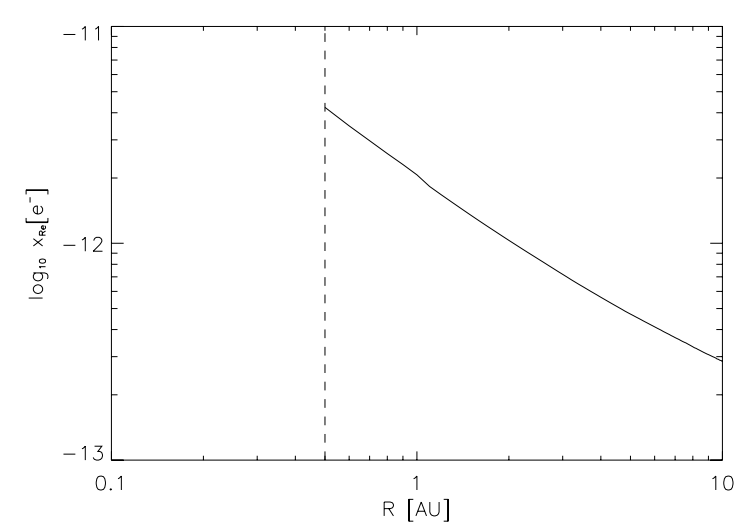

Fig. 7. The equilibrium electron concentration $x_{\mathrm{Re}}\left[\mathrm{e}^{-}\right]$for the transition layer separating the active from the dead zone vs the radial position $R$. A magnetic Reynolds number of 100 is assumed. Disk regions inside $R \leq .5 \mathrm{AU}$ are entirely active. The disk parameters are $\alpha=10^{-2}$ and $\dot{M}=10^{-7} M_{\odot} \mathrm{yr}^{-1}$.

In this study we take the value of $\mathrm{Re}_{\mathrm{m}}^{\text {crit }}=100$. For values of $\operatorname{Re}_{\mathrm{m}}$ less than this we assume that the disk is magnetically decoupled and is "dead" with respect to sustaining MHD turbulence. For $\mathrm{Re}_{\mathrm{m}} \geq 100$ we assume that the disk is turbulent. Following previous authors (e.g. Gammie 1996) we use the terms "dead" and "active" zones. We refer to the region where $\operatorname{Re}_{\mathrm{m}}=100$ separating the active and dead zones as the "transition zone". Values for the ionisation fraction $x\left[\mathrm{e}^{-}\right]$along the transition zone are given in Fig. 7, showing the values of $x\left[\mathrm{e}^{-}\right]$required to satisfy $\mathrm{Re}_{\mathrm{m}}=100$ at each radial location in the disk.

When presenting our results we typically plot the column density of the active zone and of the total disk mass as a function of cylindrical radius in the disk, rather than presenting contour plots of the free-electron abundance or magnetic Reynolds number. This is to aid comparison with results presented by Fromang et al. (2002), who also presented their results in this way, and also because it is then clear what fraction of the disk mass is active at a particular radial location in the disk.

We solved the kinetic equations by integrating over a time interval of $100000 \mathrm{yr}$. Hence, the ionisation fraction $x\left[\mathrm{e}^{-}\right]$is a function of time $t$, and in principle so is the location of the transition zone. However, for all kinetic models the change in the vertical location of the transition zone at all cylindrical radii in the computational domain was below the grid resolution for $t>10000 \mathrm{yr}$. We find that after $100000 \mathrm{yr}$, the networks model1, model2, model4 and model5 have reached chemical equilibrium. The more complex models, however, (mode13, mode16 and model7), do not reach strict chemical equilibrium in this time, although the position of the transition zone remains approximately constant. A similar situation has been found, for example, by Semenov et al. (2004), who also considered complex chemical models. These authors examined the evolution for up to $10^{6}$ years, and found that strict chemical equilibrium was still not achieved in optically thick regions.

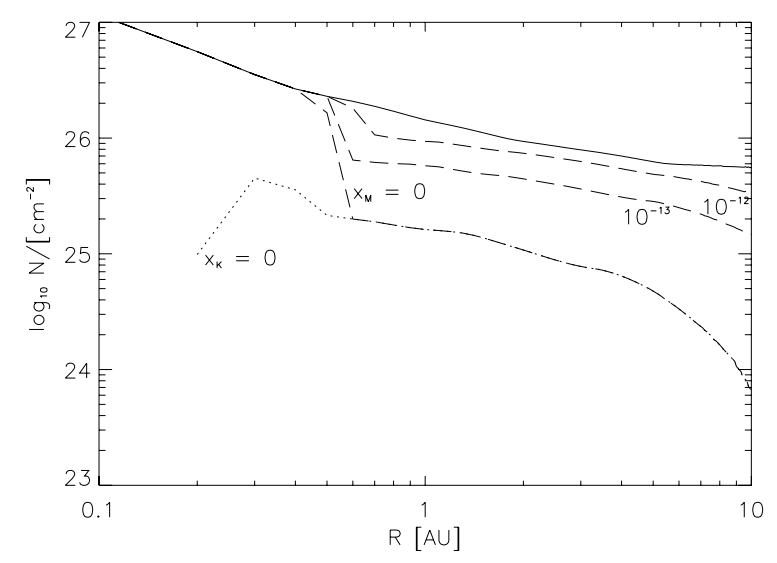

Fig. 8. Column densities of the whole disk (solid line) and of the active zones (dashed and dotted lines) - referring to magnetic Reynolds numbers greater than $100-$ for values $x_{M}=0,10^{-13}, 10^{-12}$. The dotted line refers to $x_{\mathrm{K}}=0$ (i.e. neglecting the contribution due to the thermal ionisation of potassium). The disk parameters are $\alpha=10^{-2}$ and $\dot{M}=10^{-7} M_{\odot} \mathrm{yr}^{-1}$.

\subsection{Test case}

In order to test the fidelity of our disk model and X-ray ionisation scheme we have recomputed one of the models presented in Fromang et al. (2002), who used the kinetic model of Oppenheimer \& Dalgarno (1974) to compute the ionisation fraction in $\alpha$-disk models. Equation (1) shows that the equilibrium free-electron abundance $x_{\infty}\left[\mathrm{e}^{-}\right]$depends on the effective $\mathrm{X}$-ray ionisation rate $\zeta_{\mathrm{eff}}(r, z)$, the number density of the neutral particles $n$, and the gas temperature $T$. We calculated the electron fraction using the same parameter values used by Fromang et al. (2002), and obtained the spatial distribution of $\operatorname{Re}_{\mathrm{m}}$ using Eqs. (4) and (5). We plot the column densities of the active zone obtained by our model in Fig. 8, which should be compared with Figs. 4 and 5 in Fromang et al. (2002). The agreement is excellent.

For high temperature (and density) regions, we have considered the contributions of ground state ionized atoms under the assumption of local thermodynamic equilibrium. Despite their low abundances, potassium $(\mathrm{K})$ and sodium $(\mathrm{Na})$ provide major contributions the ionisation fraction due to the small values of the first ionisation potentials, 5.14 and $4.34 \mathrm{eV}$, respectively.

Applying Eq. (1) of Fromang et al. (2002), we considered the contribution of thermal ionisation of potassium to the electron abundance. Neglecting this particular contribution leads to smaller values in the column densities of the active zone in the very inner regions only as shown in Fig. 8. Including it ensures that the whole disk within about $0.4 \mathrm{AU}$ is active.

\subsection{Gas-phase chemistry}

\section{model1}

In Sect. 5.1 the electron fraction predicted by the Oppenheimer \& Dalgarno (1974) kinetic model was obtained using an analytic approximation, giving rise to the results in Fig. 8. We have also solved the full kinetic equations of model1, and can 


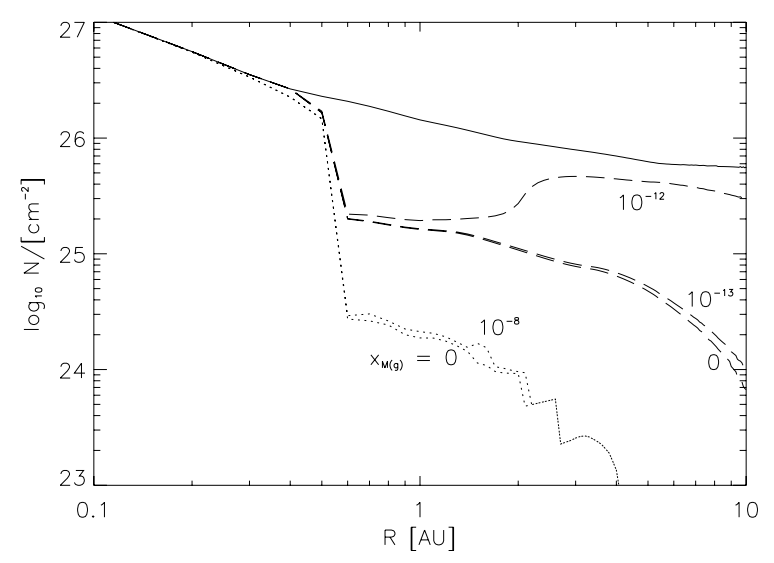

Fig. 9. model 1/model 4 - Column densities of the whole disk (solid line) and of the active zones (dashed and dotted lines) - referring to magnetic Reynolds numbers greater than 100 - for different values $x_{\mathrm{M}(\mathrm{g})}$. While the dashed lines refer to a value $x_{\mathrm{gr}}=0$ (gas-phase $\mathrm{ch}$.), the dotted lines refer to a value $x_{\mathrm{gr}}=10^{-12}$ (gas-grain ch.). Especially, for a grain free environment no dead zones are observed above values $x_{\mathrm{M}} \geq 10^{-11}$. The disk parameters are $\alpha=10^{-2}$ and $\dot{M}=10^{-7} M_{\odot} \mathrm{yr}^{-1}$.

compare the exact equilibrium value of $x\left[\mathrm{e}^{-}\right]$with the equilibrium value $x_{\infty}\left[\mathrm{e}^{-}\right]$given by Eq. (1).

We find that the solution for $x_{\infty}\left[\mathrm{e}^{-}\right]$obtained from Eq. (1) is very accurate if the exact equilibrium values for neutral metal atoms, $x_{\mathrm{M}}$, is used in Eq. (1). Comparing our results in Fig. 9 to those presented in Fig. 8 and Fromang et al. (2002), we obtain agreement when the metal abundance $x_{\mathrm{M}}=0$. We find significant differences, however, when the metal abundance is $x_{\mathrm{M}}=10^{-13}$ or $10^{-12}$. The differences can be traced to the assumption, used to obtain Fig. 8, that the equilibrium concentration of neutral metals, $x_{\infty}[\mathrm{M}]$, is constant and equal to the total fractional abundance of metals. In fact the full solution to the kinetic equations predicts that $x[\mathrm{M}]$ can vary by up to two orders of magnitude, especially within $R \leq 2 \mathrm{AU}$, where the effects of the metals in increasing the vertical size of the active zone is correspondingly diminished.

As already discussed at some length by Fromang et al. (2002), the addition of small quantities of metals (much below solar abundance) to the reaction scheme causes the predicted size of the active zone to increase. This is because the chargetransfer reaction between the metal and molecular ion reduces the concentration of molecular ions which recombine with electrons rapidly. The recombination rate between metal ions and electrons is much smaller, leading to a higher equilibrium electron fraction. Comparing the rate coefficient for molecular ion-electron recombination $(\tilde{\alpha})$ with that for metal ion-electron recombination $(\tilde{\gamma})$ illustrates this point (see Table 1 ).

Moving out from the centre of the disk, the features observed in the size of the active zone can be explained as follows. Interior to $R \leq 0.5 \mathrm{AU}$ the disk is completely active due to thermal ionisation of potassium. For values of $x_{\mathrm{M}}=10^{-13}$ and $10^{-12}$ the region between $0.5 \leq R \leq 2$ AU maintains a deep dead zone because the metal concentration is too small to prevent $\mathrm{H}_{2}^{+}$being the dominant ion, whose continued presence maintains a low electron fraction. Exterior to $2 \mathrm{AU}$ a metal concentration significantly enlarges the active zone. Primarily this

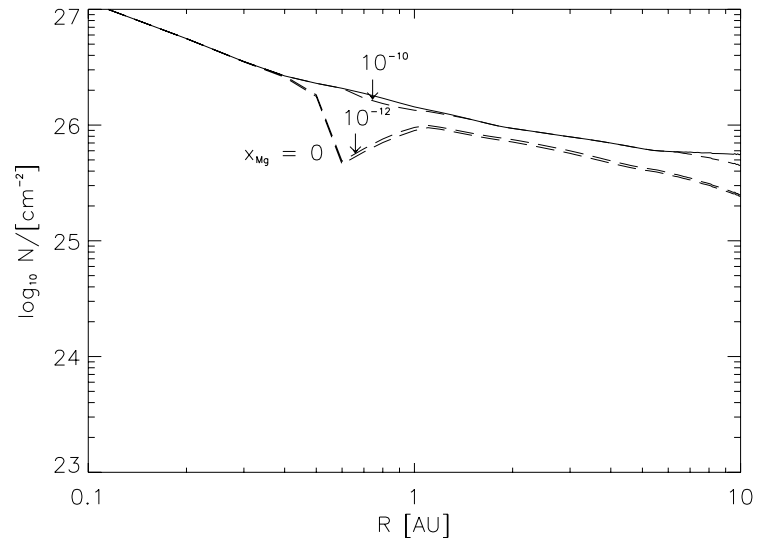

Fig. 10. model 2 - Column densities of the whole disk (solid line) and of the active zones (dashed lines) - referring to magnetic Reynolds numbers greater than 100 - for values $x_{\mathrm{Mg}}=0,10^{-12}, 10^{-10}$. The disk parameters are $\alpha=10^{-2}$ and $\dot{M}=10^{-7} M_{\odot} \mathrm{yr}^{-1}$.

is because a lower ionisation degree is required at larger radius to obtain an active layer with $\operatorname{Re}_{\mathrm{m}} \geq 100$, as shown by Fig. 7 . A relatively low abundance of metals is required to achieve this threshold at radii beyond 2 AU. Note that for metal concentrations $x_{\mathrm{M}} \geq 10^{-11}$ the whole of our disk model is active.

\section{model2}

This model takes the elements hydrogen and magnesium, and extracts all the relevant species and reactions from the UMIST database. Solving the resulting reaction set for model2 produces an active zone with much larger column density than obtained using model1. This is illustrated by comparing Figs. 9 and 10. It is clear that this effect has nothing to do with the presence of metals, as the active zone is large in their absence. Instead the hydrogen chemistry contained in the UMIST database leads to a dominant molecular ion $\mathrm{H}^{+}$whose recombination time is much longer than that $\left(\right.$ for $\mathrm{H}_{2}^{+}$) in the Oppenheimer \& Dalgarno model. The active zone predicted by model2 is also much larger than predicted by a much more complex gas-phase chemistry (model3) so we neglect it from further discussion as it seems to be unreliable.

\section{model3}

This model was constructed by extracting all species and reactions from Table A. 1 and the UMIST database containing the elements $\mathrm{H}, \mathrm{He}, \mathrm{C}, \mathrm{O}, \mathrm{N}, \mathrm{S}, \mathrm{Si}, \mathrm{Mg}$, Fe. The results obtained when solving the model 3 reaction set are shown in Fig. 11. Comparing this with Fig. 9, it is apparent that model3 predicts smaller active zones than model1, both with and without metals. The metal atoms included in model 3 were magnesium and iron, however we find essentially no difference in the ionisation structure of the disk when iron is neglected.

Apart from the chemiionisation reaction discussed in Sect. 4, free electrons can only be created by the photoionisation of $\mathrm{H}_{2}$ and $\mathrm{He}$. The inclusion of He thus increases the ionisation rate compared to that in model1. Removal of free electrons occurs primarily through dissociative recombination reactions with ions. Most of these ions are formed through charge transfer-reactions that are initiated by the 


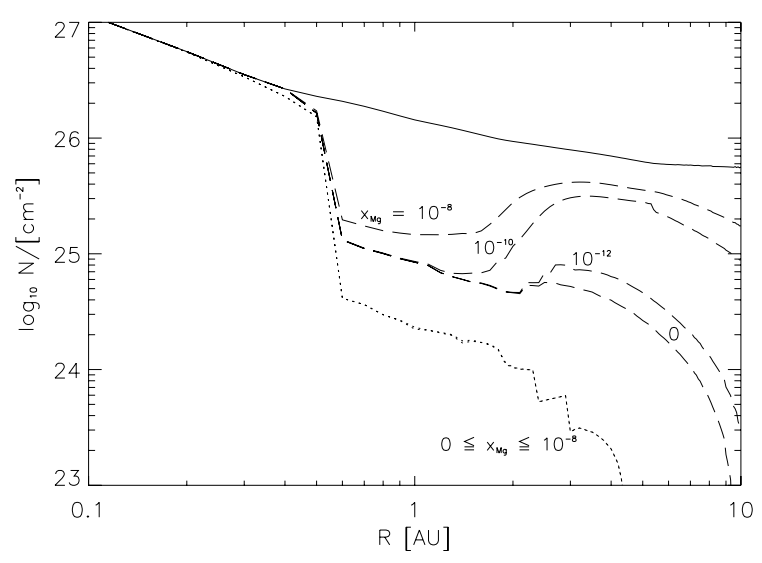

Fig. 11. model 3/model7 - Column densities of the whole disk (solid line) and of the active zones (dashed and dotted lines) - referring to magnetic Reynolds numbers greater than 100 - for values $x_{\mathrm{Mg}}=$ $0,10^{-12}, 10^{-10}, 10^{-8}\left(x_{\mathrm{Fe}}=0\right)$. The dashed lines refer to a value $x_{\mathrm{gr}}=0$ (gas-phase ch.), while the dotted lines refer to $x_{\mathrm{gr}}=10^{-12}$ (gas-grain ch.). The disk parameters are $\alpha=10^{-2}$ and $\dot{M}=10^{-7} M_{\odot} \mathrm{yr}^{-1}$.

photoionisation of hydrogen and helium. This leads to the formation of about 100 ionized gas-phase species in model 3 which may recombine with electrons.

For $x_{\mathrm{Mg}}=0$, the only ion in model1 is $\mathrm{H}_{2}^{+}$. In model3 the dominant molecular ions are $\mathrm{HCNH}^{+}, \mathrm{H}_{4} \mathrm{C}_{2} \mathrm{~N}^{+}, \mathrm{CNC}^{+}$and $\mathrm{NO}^{+}$. Although their concentration values are below the value of $x\left[\mathrm{H}_{2}^{+}\right]$obtained using model1, they destroy $\mathrm{e}^{-}$more efficiently than $\mathrm{H}_{2}^{+}$.

For $x_{\mathrm{Mg}} \neq 0, \mathrm{Mg}^{+}$becomes by far the most dominant ion in the active zone as $x_{\mathrm{Mg}}$ is increased. However, due to the much larger rate coefficients for dissociative recombination reactions involving ions such as $\mathrm{NH}_{4}^{+}, \mathrm{NO}^{+}$and $\mathrm{HCNH}^{+}$, these more complex species remain the dominant species that recombine with free electrons. Hence, $\mathrm{Mg}^{+}$ions change rather than dominate the destruction rate for free electrons. This remains the case even for relatively large metal abundances up to $x_{\mathrm{Mg}}=10^{-8}$, such that a dead zone remains for this value.

Motivated by the results of Sano et al. (2000) and those obtained using model3 which show the nitrogen bearing species to be important, we studied the effect of nitrogen on the ionisation fraction by implementing model6, which does not include nitrogen bearing species. We considered the gas-phase kinetics of model 6 by setting $x_{\mathrm{gr}}=0$. The molecular ions which dominate the destruction of $\mathrm{e}^{-}$now differ from those in model3. Dissociative recombination reactions with ions such as $\mathrm{H}_{3} \mathrm{O}^{+}$, $\mathrm{HCO}^{+}$(for $x_{\mathrm{Mg}}=0$ ) and $\mathrm{Mg}^{+}, \mathrm{H}_{3} \mathrm{O}^{+}, \mathrm{C}_{3} \mathrm{H}_{3}^{+}$and $\mathrm{CH}_{3} \mathrm{CO}^{+}$(for $x_{\mathrm{Mg}} \neq 0$ ) dominate the destruction process of $\mathrm{e}^{-}$. However, we find that the location of the transition zone is only significantly modified in the regions interior to $R=2 \mathrm{AU}$, as may be observed when comparing Figs. 11 and 12. The neglect of nitrogen bearing species increases the size of the active zone, indicating that these species are important in determining the ionisation balance in protostellar disks. However, it is also clear that the remaining non-nitrogen bearing molecular ions are equally important, and lead to the formation of a smaller active zone than predicted by the simple Oppenheimer \& Dalgarno (1974) model. Even a metal abundance of $x_{\mathrm{Mg}}=10^{-8}$ is

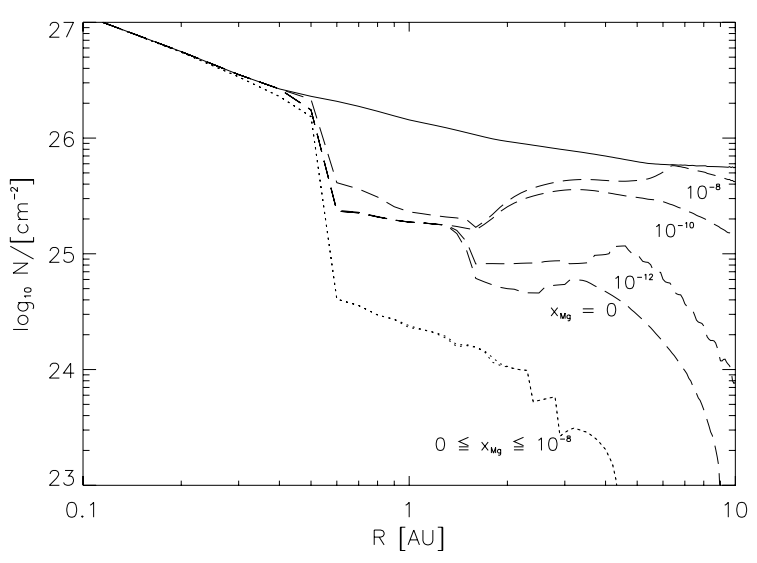

Fig. 12. model6 - Column densities of the whole disk (solid line) and of the active zones (dashed and dotted lines) - referring to magnetic Reynolds numbers greater than 100 - for values $x_{\mathrm{Mg}}=$ $0,10^{-12}, 10^{-10}, 10^{-8}$. The dashed lines refer to a value $x_{\mathrm{gr}}=0$ (gasphase $\mathrm{ch}$.), while the dotted lines refer to $x_{\mathrm{gr}}=10^{-12}$ (gas-grain ch.). The disk parameters are $\alpha=10^{-2}$ and $\dot{M}=10^{-7} M_{\odot} \mathrm{yr}^{-1}$.

insufficient to make the disk fully active due to the continued dominance of the molecular ions.

We conclude that the additional molecular ions generated in more complex reaction networks generally lead to a smaller fractional abundance of free electrons than the simple Oppenheimer \& Dalgarno (1974) reaction scheme. Hence a larger dead zone occurs within protostellar disks than is predicted using the simple scheme in conjunction with the rate coefficients listed in Table 1. However, in Sect. 6 we show that by making modest changes to the rate coefficients in the Oppenheimer \& Dalgarno model good agreement may be obtained with the full UMIST database model for certain metal abundances.

\subsection{Gas-grain chemistry}

We now present results from kinetic models that include gasphase reactions, "mantle chemistry", and "grain chemistry" (see Sect. 3.1.3 for a definition of these terms). We consider grains of a fixed size $\left(r_{\mathrm{gr}}=10^{-5} \mathrm{~cm}\right)$ and with the possibility of having five different charges: $\mathrm{gr} \mathrm{gr}^{ \pm}$, and $\mathrm{gr}^{2 \pm}$. Our motivation for examining these models is to first address the question of how the various gas-phase models change and differ from one another when grains are introduced. Second, to examine the effects of depleting the concentration of small grains on the chemistry as a crude means of modelling the effects of grain growth, and third to define under which conditions gas-grain chemistry allows the existence of a significant active zone.

\section{model4/model5}

The first reaction scheme including gas-grain chemistry used to examine the issue of the ionisation fraction in protostellar disks was introduced by Sano et al. (2000). Their model is equivalent to our mode15. We begin our discussion by first examining the gas-phase chemistry produced by model5 (i.e. with $x_{\mathrm{gr}}=0$ ). The results are shown in Fig. 13. Comparing Fig. 13 with Fig. 8, we find that model 5 is in close agreement with 


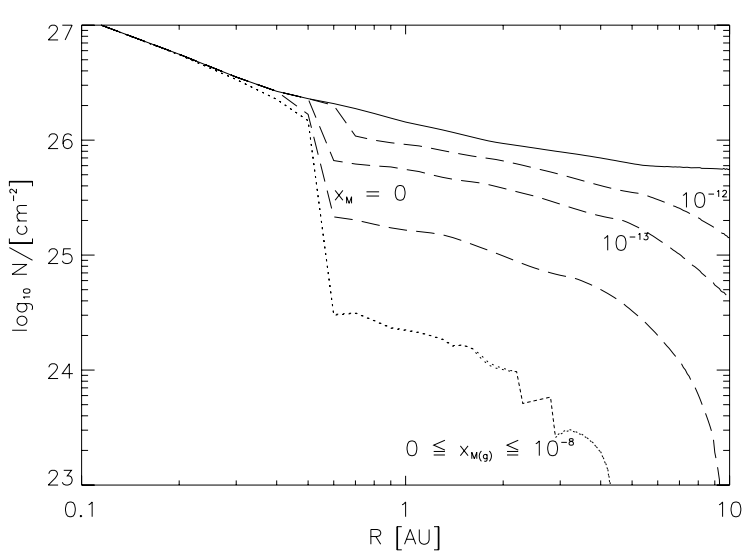

Fig. 13. model 5 - Column densities of the whole disk (solid line) and of the active zones (dashed and dotted lines) - referring to magnetic Reynolds numbers greater than 100 - for different values of $x_{\mathrm{M}(\mathrm{g})}$. While the dashed lines refer to $x_{\mathrm{gr}}=0$ (gas-phase ch.), the dotted lines refer to $x_{\mathrm{gr}}=10^{-12}$ (gas-grain ch.). The disk parameters are $\alpha=10^{-2}$ and $\dot{M}=10^{-7} M_{\odot} \mathrm{yr}^{-1}$.

model1 (i.e. the Oppenheimer \& Dalgarno model for which $x\left[\mathrm{e}^{-}\right]$is calculated from Eq. (1)). We note that the introduction of grain chemistry dramatically decreases the size of the active zone, and gives agreement with model 4 .

We now discuss the results obtained with model4. This model is an extension of the Oppenheimer \& Dalgarno (1974) reaction scheme (model1) which includes gas-grain interactions. The original model included a generalised molecule and a generalised metal. As we need to specify the evaporation temperature from the grains for these components, we use the species $\mathrm{H}_{2}$ and $\mathrm{Mg}$.

The results obtained with model4, but with magnesium not being included, are presented in Fig. 14 (we discuss the results with magnesium included below). In the limit of $x_{\mathrm{gr}}=0$ this model reduces to model 1 . The introduction of grains with concentration $x_{\mathrm{gr}}=10^{-12}$ leads to a dramatic reduction in the size of the active zone, as the grains are very efficient at sweeping up most of the free electrons. This result is in basic agreement with expectations.

We now consider the effect of reducing the grain concentration. Figure 14 shows the effect of including grains with concentrations $x_{\mathrm{gr}}=10^{-14}$ and $10^{-16}$. A grain concentration of $x_{\mathrm{gr}}=10^{-16}$ is required for the gas-grain chemistry to generate an active zone that is the same size as the pure gas-phase chemistry. This result is interesting, and at first sight rather puzzling, since Fig. 7 shows that the electron fraction required for the disk to be active is between $x\left[\mathrm{e}^{-}\right]=3 \times 10^{-13}$ and $4 \times 10^{-12}$. If the grains simply act as a means of sweeping up the free electrons, how can a very small concentration of grains such as $x_{\mathrm{gr}}=10^{-14}$ significantly affect the position of the transition between active and dead zones?

In the simple model 4 without magnesium, the grain particles are involved in the main destruction paths for $\mathrm{H}_{2}^{+}$and $\mathrm{e}^{-}$. While free electrons are mostly destroyed by neutral grains through the reaction

$\mathrm{e}^{-}+\mathrm{gr} \longrightarrow \mathrm{gr}^{-}$,

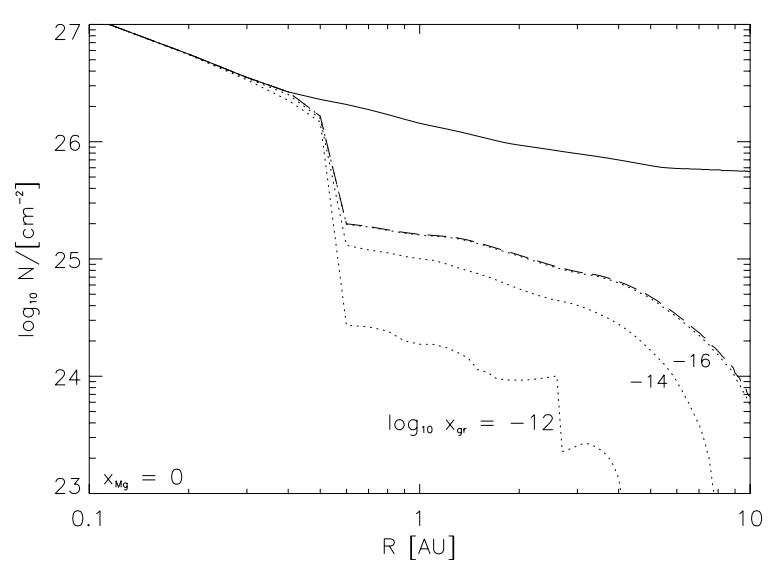

Fig. 14. model 4 - Column densities of the whole disk (solid line) and of the active zones (dashed and dotted lines) - referring to magnetic Reynolds numbers greater than 100 - for different values of $x_{\mathrm{gr}}$ (while $x_{\mathrm{Mg}}=0$ is assumed). While the dashed line refers to $x_{\mathrm{gr}}=0$ (gasphase ch.), the dotted lines refer to $x_{\mathrm{gr}} \neq 0$ (gas-grain ch.). The disk parameters are $\alpha=10^{-2}$ and $\dot{M}=10^{-7} M_{\odot} \mathrm{yr}^{-1}$.

$\mathrm{H}_{2}^{+}$is destroyed by recombination with singly negatively charged grain particles

$\mathrm{H}_{2}^{+}+\mathrm{gr}^{-} \longrightarrow \mathrm{H}_{2}+\mathrm{gr}$.

These two processes form a loop that recycles neutral grain particles, ensuring that they always participate actively in the chemical evolution. The equilibrium electron fraction $x_{\infty}\left[\mathrm{e}^{-}\right]$ is determined by a balance between production and destruction processes. Apparently only a small number of active neutral grains are required to modify the equilibrium ionisation structure in a disk due to the high rate with which electrons accrete onto the grains.

In order to reduce the destruction of $\mathrm{e}^{-}$by neutral grains one may limit the neutral grain concentration $x[\mathrm{gr}]$ by:

(i) reducing $x_{\mathrm{gr}}$ - the total grain concentration;

(ii) limit the formation of neutral grains by breaking the cycle described above.

Satisfying option $(i)$ requires the value $x_{\mathrm{gr}}=10^{-16}$ already discussed. By contrast, we find that the transition from gas-grain dominated chemistry to gas-phase chemistry can be achieved for values $x_{\mathrm{gr}} \sim 10^{-14}$ if the cycle is short-circuited by setting the rate coefficient of the reaction $\mathrm{H}_{2}^{+}+\mathrm{gr}^{-} \longrightarrow \mathrm{H}_{2}+$ gr to zero (i.e. by preventing the neutralisation of negative grains). Preventing the conversion of $\mathrm{gr}^{2-}$ to $\mathrm{gr}^{-}$by a similar reaction allows a grain concentration of $x_{\mathrm{gr}} \simeq 10^{-13}$ to reproduce the gas-phase chemistry. This is because all the neutral grains become and remain negatively charged, after which they no longer participate in the chemical evolution.

We now discuss the results of model 4 with magnesium being included with concentration $x_{\mathrm{Mg}}=10^{-12}$. These calculations are presented in Fig. 15. The grains are very efficient at sweeping up magnesium, and thermal desorption from the grain surfaces is rather inefficient. The result is that the active zone with grain concentration $x_{\mathrm{gr}}=10^{-12}$ and magnesium concentration $x_{\mathrm{Mg}}=10^{-12}$ is almost indistinguishable from the case with $x_{\mathrm{Mg}}=0$ (see Fig. 9). 


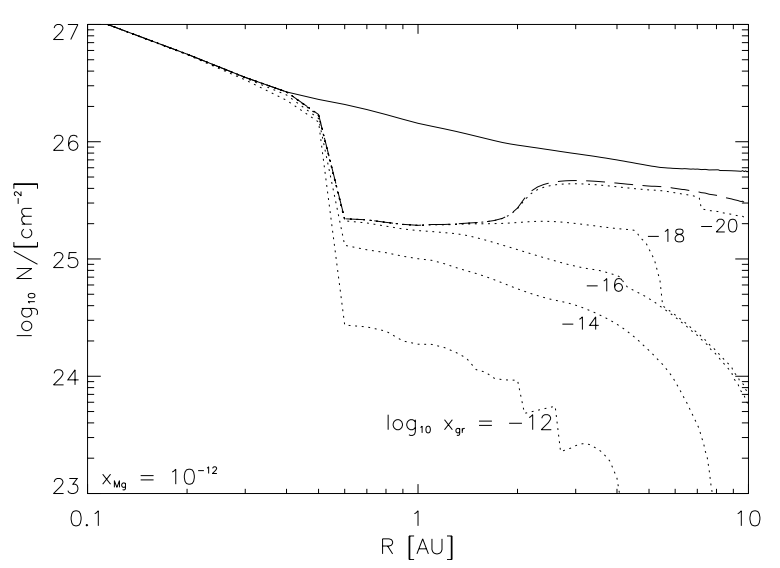

Fig. 15. model 4 - Column densities of the whole disk (solid line) and of the active zones (dashed and dotted lines) - referring to magnetic Reynolds numbers greater than 100 - for different values $x_{\mathrm{gr}}\left(x_{\mathrm{Mg}}=\right.$ $10^{-12}$ is assumed). While the dashed line refers to a value $x_{\mathrm{gr}}=0$ (gasphase ch.), the dotted lines refer to $x_{\mathrm{gr}} \neq 0$ (gas-grain ch.). The disk parameters are $\alpha=10^{-2}$ and $\dot{M}=10^{-7} M_{\odot} \mathrm{yr}^{-1}$.

A series of runs were performed to examine the effect of reducing the grain concentration $x_{\mathrm{gr}}$. In particular we are interested in defining the grain concentration required to reproduce the pure gas-phase chemistry of model1 with $x_{\mathrm{Mg}}=10^{-12}$. The results of these calculations are presented in Fig. 15. Reducing $x_{\mathrm{gr}}$ to $10^{-16}$ results in an active zone whose size is the same as that produced by the pure gas-phase chemistry (model1) but with $x_{\mathrm{Mg}}=0$. In order to obtain an active zone that is the same size as that obtained with the gas-phase chemistry (model1) with $x_{\mathrm{Mg}}=10^{-12}$ we had to reduce the grain concentration by more than eight orders of magnitude to $x_{\mathrm{gr}}=10^{-21}-10^{-20}$. The reasons for this are similar to those already discussed for mode14 with $x_{\mathrm{Mg}}=0$. The equilibrium abundance of free electrons is determined by a balance between production and destruction processes. Production occurs through photoionisation of $\mathrm{H}_{2}$ only. Destruction is dominated by the reaction $\mathrm{gr}+\mathrm{e}^{-} \rightarrow \mathrm{gr}^{-}$. Only a very small number of neutral grains are required to reduce the equilibrium electron fraction below that obtained by the gas-phase chemistry. When $\mathrm{Mg}$ is included in the model, the gas-phase chemistry produces a larger electron fraction than when $x_{\mathrm{Mg}}=0$. Obtaining this enhanced electron fraction means that the required depletion of grains is greater when $x_{\mathrm{Mg}} \neq 0$.

Neutral grains are recycled in this case by the reaction $\mathrm{gr}^{-}+\mathrm{Mg}^{+} \rightarrow \mathrm{gr}+\mathrm{Mg}$ as $\mathrm{Mg}^{+}$is the dominant gas-phase ion. By switching off this reaction (and the counterpart for $\mathrm{gr}^{2-}+\mathrm{Mg}^{+}$) in model4, and all reactions involving the adsorption of magnesium onto grains, we obtained an active zone whose size was the same as that obtained by the pure gas-phase chemistry (model1), but now with $x_{\mathrm{gr}}=10^{-16}$ rather than $10^{-21}$.

Simply switching off adsorption of magnesium onto grains, but continuing to allow $\mathrm{Mg}^{+}$to neutralise negatively charged grains $\mathrm{gr}^{-}$resulted in a value of $x_{\mathrm{gr}}=10^{-20}$ being required to reproduced the gas-phase chemistry. Thus the production of a sizeable active zone in the presence of small grains clearly requires either a highly diminished population of these small grains, or a means of preventing negatively charged grains from being continuously neutralised by positive ions.

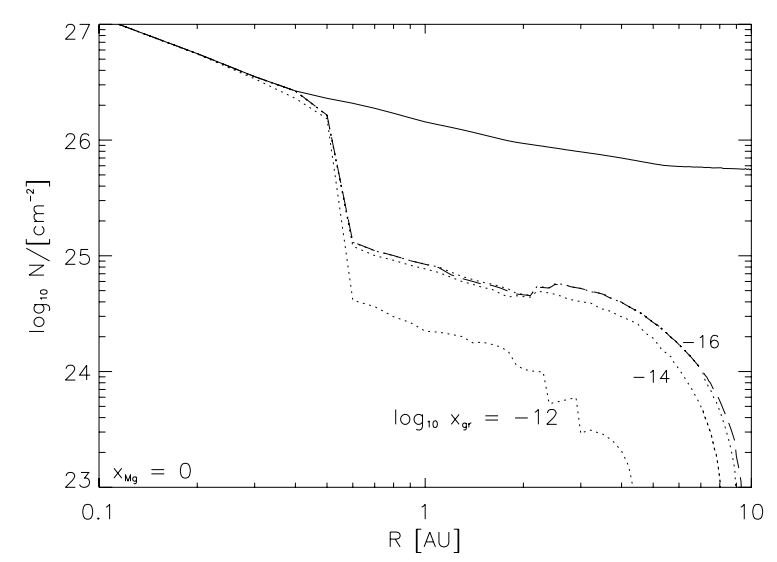

Fig. 16. model 7 - Column densities of the whole disk (solid line) and of the active zones (dashed and dotted lines) - referring to magnetic Reynolds numbers greater than 100 - for different values of $x_{\mathrm{gr}}$ (while $x_{\mathrm{Fe}}=x_{\mathrm{Mg}}=0$ is assumed). While the dashed line refers to $x_{\mathrm{gr}}=0$ (gas-phase ch.), the dotted lines refer to $x_{\mathrm{gr}} \neq 0$ (gas-grain ch.). The disk parameters are $\alpha=10^{-2}$ and $\dot{M}=10^{-7} M_{\odot} \mathrm{yr}^{-1}$.

\section{model6/model7}

We now discuss the results obtained with model 6 and model7. We remind the reader that model 6 was constructed by examining the elements contained in the Sano et al. (2000) model (equivalent to our mode15), and extracting all species from Table A.1. All reactions of the UMIST database are considered that involve these species. model7 was constructed similarly by specifying a larger set of elements $(\mathrm{H}, \mathrm{He}, \mathrm{C}, \mathrm{N}$. O, S, Si, $\mathrm{Mg}, \mathrm{Fe}$ ).

As a general result we find that the location of the transition zone is very similar when comparing model4, model 6 and model 7 when we use a standard grain concentration of $x_{\mathrm{gr}}=10^{-12}$. This shows that the grain chemistry is dominant in determining the ionisation fraction. Given this fact we do not discuss model 6 any further.

We first consider model7 when magnesium is neglected from the model. Results for this case are shown in Fig. 16 for a variety of chosen grain concentrations. We will discuss cases with $x_{\mathrm{Mg}} \neq 0$ later on in this section.

For grain concentration $x_{\mathrm{gr}}=10^{-12}$ the dead zone is significantly larger than obtained with the pure gas-phase equivalent mode13. Decreasing the grain concentration to $x_{\mathrm{gr}}=10^{-16} \mathrm{re}-$ sulted in an active zone which is the same size as obtained by the pure gas-phase model (model3). The reasons for this were discussed at length when describing the results from model4, and we do not repeat them here. We have run numerical experiments in which singly negative-charged grains are prevented from neutralising via recombination reactions with positive ions. In these models a grain concentration of $x_{\mathrm{gr}} \simeq 10^{-13}$ was required to reproduce the size of the active zone obtained in the equivalent pure gas-phase model (model3).

We now discuss results obtained using model7 with $x_{\mathrm{Mg}}=$ $10^{-8}$. These are presented in Fig. 17. For a grain concentration of $x_{\mathrm{gr}}=10^{-12}$ we find that the active zone is almost identical to that obtained when $x_{\mathrm{Mg}}=0$. This is because the grains are very effective at sweeping up the metal atoms and free electrons. As with model4, a reduction of the grain concentration 


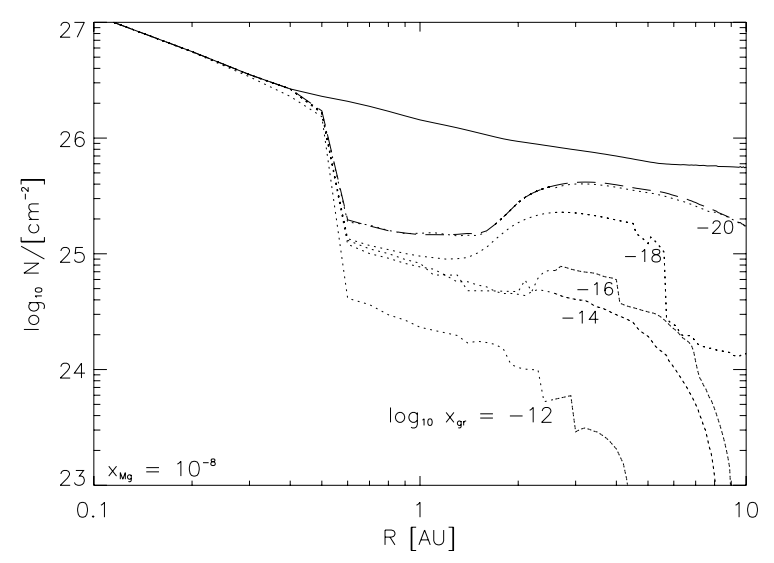

Fig. 17. model 7 - Column densities of the whole disk (solid line) and of the active zones (dashed and dotted lines) - referring to magnetic Reynolds numbers greater than 100 - for different values of $x_{\mathrm{gr}}$ (while $x_{\mathrm{Fe}}=0, x_{\mathrm{Mg}}=10^{-8}$ is assumed). While the dashed line refers to $x_{\mathrm{gr}}=0$ (gas-phase ch.), the dotted lines refer to $x_{\mathrm{gr}} \neq 0$ (gas-grain ch.). The disk parameters are $\alpha=10^{-2}$ and $\dot{M}=10^{-7} M_{\odot} \mathrm{yr}^{-1}$.

to $x_{\mathrm{gr}}=10^{-16}$ leads to an active zone whose size is very similar to that obtained using a pure gas-phase chemistry without metals (model3). A further reduction is grain concentration to $x_{\mathrm{gr}}=10^{-20}$ is required to obtain an active zone of the same size as obtained by pure gas-phase chemistry with magnesium abundance $x_{\mathrm{Mg}}=10^{-8}$. Once again the reasons are the same as described for model4, and we do not repeat them here. However, it is worth re-emphasising the fact that the active zone obtained by the pure gas-phase chemistry of model 3 can be obtained for a smaller grain depletion factor $\left(x_{\mathrm{gr}}=10^{-16}\right)$ if singly-negative charged grains are prevented from forming neutral grains via recombination reactions with positive ions.

\section{Discussion}

We now discuss issues relating to potential omissions from and extensions to the kinetic models presented in Sect. 5. Apart from the model discussed in "Fitting the $x\left[\mathrm{e}^{-}\right]$distribution" all other models we discuss in this section assumed $x_{\mathrm{Mg}}=$ $1.09 \times 10^{-8}$ and $x_{\mathrm{Fe}}=2.74 \times 10^{-9}$ instead of $x_{\mathrm{Mg}}=$ variable and $x_{\mathrm{Fe}}=0$.

\section{RADIONUCLIDES}

Sano et al. (2000) and Semenov et al. (2004) included ionisation due to the decay of radioisotopes in their models. We have run models with this included, using a conservative estimate of the ionisation rate of $\zeta_{\mathrm{R}} \approx 6.9 \times 10^{-23} \mathrm{~s}^{-1}$ following Sano et al. (2000). We find that this is not an important source of ionisation in our models.

\section{NONTHERMAL DESORPTION PROCESSES}

Najita et al. (2001) examined the potential effect of nonthermal desorption of grain mantles by X-ray irradiation. We have investigated this effect in the simplest possible way, by switching off all reactions that involve the adsorption of species onto grain surfaces. It should be noted, however, that we still allow electrons to accrete onto grain surfaces at the usual rates. We re-ran

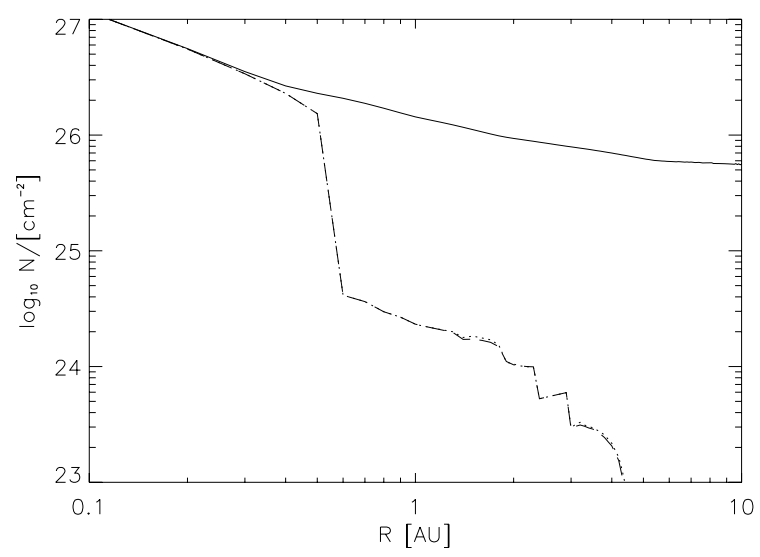

Fig. 18. model 7 - Column densities of the whole disk (solid line) and of the active zones (dotted and dashed lines) - referring to magnetic Reynolds numbers greater than 100 . While the dashed line refers to simulation which takes account of all types of reactions, the dotted line refers to a simulation where all reactions which may form a mantle species were neglected. The assumed metal abundances are: $x_{\mathrm{Fe}}=2.74 \times 10^{-9}$ and $x_{\mathrm{Mg}}=1.09 \times 10^{-8}$ while $x_{\mathrm{gr}}=10^{-12}$. The disk parameters are $\alpha=10^{-2}$ and $\dot{M}=10^{-7} M_{\odot} \mathrm{yr}^{-1}$.

model7 with this modification to the reaction network. The results are shown in Fig. 18, demonstrating clearly that the adsorption of molecular, atomic, and ionic species onto grain surfaces plays essentially no role in determining the size of the active zone.

\section{FITTING THE $x\left[\mathrm{e}^{-}\right]$DISTRIBUTION}

In Sect. 5 we computed a variety of kinetic models for the primary purpose of examining the size of the active zone. Considering first the gas-phase chemistry, we found significant differences in the predictions of the simplest model (model1) and the most complex model (model3). Future research in the dynamics of protoplanetary disks will eventually involve a direct coupling between magnetohydrodynamic simulations of turbulent disks, and chemical networks that self-consistently calculate the ionisation fraction. The task of coupling these two approaches would be greatly simplified if a chemical network containing few species could be developed that calculates the ionisation fraction accurately. For this reason we are motivated to examine if simple changes to the rate coefficients in the Oppenheimer \& Dalgarno (1974) network (model1) can lead to agreement with the predicted ionisation fraction given by the complex UMIST-based model (mode13).

The results of our attempts to fit model 3 by modifying model 1 is shown in Fig. 19. For a metal abundance $x_{\mathrm{Mg}}=$ $10^{-12}$ we obtain a good fit to the size of the active zone by increasing the rate coefficients $\tilde{\alpha}$ and $\tilde{\gamma}$ by factors of 10 and 200 compared to the values given in Table 1 . The spatial distribution of the electron fraction obtained using model 3 is shown in Fig. 20 and that obtained using the modified model 1 is shown in Fig. 21. Although there are small differences, overall these distributions agree well.

The agreement is not so good when we increase the metal abundance to $x_{\mathrm{Mg}}=10^{-10}$. We found that simple modifications to $\tilde{\alpha}$ and $\tilde{\gamma}$ were unable to produce a good overall fit. One is 


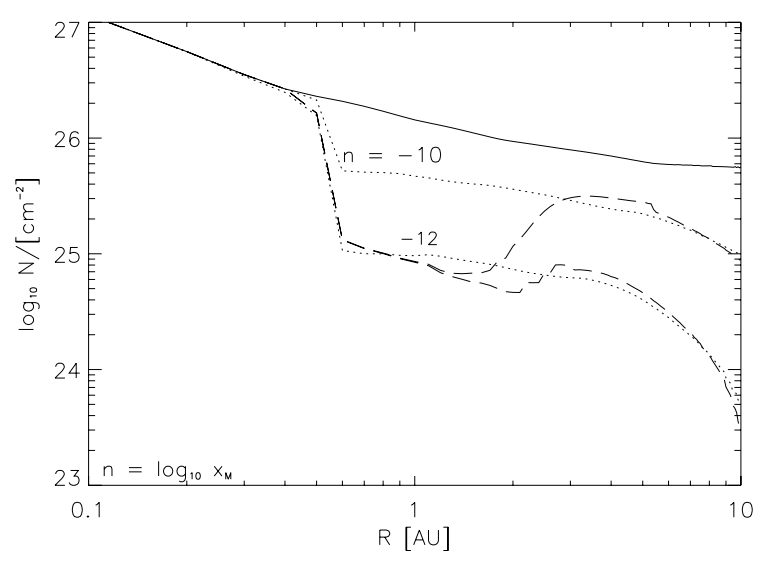

Fig. 19. Column densities of the whole disk (solid line) and of the active zones (dashed and dotted lines) - referring to magnetic Reynolds numbers greater than 100 - for two different kinetic models of gasphase chemistry with $x_{\mathrm{Mg}}=10^{-12}, 10^{-10}$. The dashed line refers to the UMIST model (model3) while the dotted line refers to the Oppenheimer \& Dalgarno model (model1) with modified rate coefficients $\tilde{\alpha}$ and $\tilde{\gamma}$. Compared with the values in Table $1, \tilde{\alpha}$ and $\tilde{\gamma}$ are increase by 10 and 200, respectively. The disk parameters are $\alpha=10^{-2}$ and $\dot{M}=10^{-7} M_{\odot} \mathrm{yr}^{-1}$.

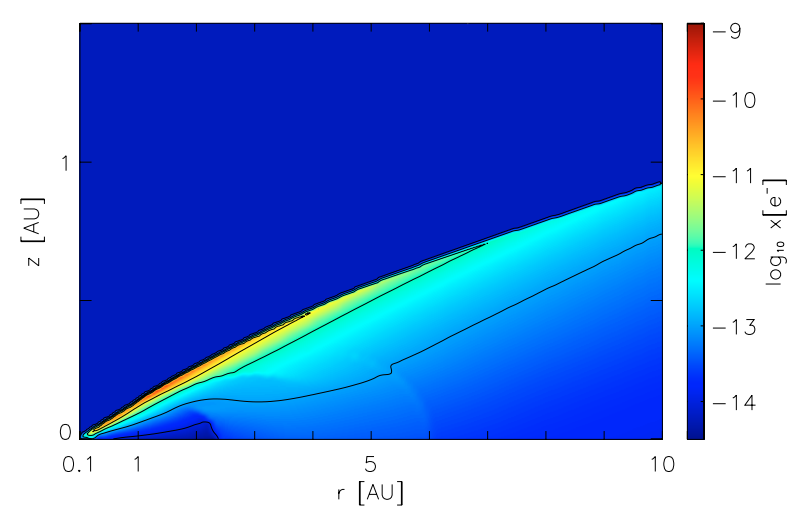

Fig. 20. This contour plot shows the electron distribution at $t=10^{5} \mathrm{yrs}$ for our $\alpha=10^{-2}, \dot{M}=10^{-7} M_{\odot} \mathrm{yr}^{-1}$ disk model by applying the UMIST model (model3) with $x_{\mathrm{Mg}}=10^{-12}$. The contour lines refer to values $x\left[\mathrm{e}^{-}\right]$of $10^{-14}, 10^{-13}, 10^{-12}$, and $10^{-11}$.

able to fit either the inner $(R \leq 2 \mathrm{AU})$ or outer region $(R \geq$ 2 AU) quite well, but not both at the same time (Fig. 19 shows a case where we have fitted the outer region). This is because the chemistry in model 1 is dominated by the metal ion when $x_{\mathrm{Mg}}>10^{-12}$, whereas this is not the case for model3.

The situation with the gas-grain chemistry is much more simple. For a standard abundance of submicron sized grains, the simple model (model4) and the complex model (model7) are in extremely good agreement.

\section{Disk Model}

In addition to computing the ionisation fraction in disk models with $\alpha=10^{-2}$ and $\dot{M}=10^{-7} M_{\odot} \mathrm{yr}^{-1}$, we also computed some cases for which the disk parameters are $\alpha=5 \times 10^{-3}$ and $\dot{M}=10^{-8} M_{\odot} \mathrm{yr}^{-1}$. It may be argued that these are closer to the canonical values for T Tauri stars. The effective X-ray ionisation rate for this model is shown in Fig. 22. The lower mass

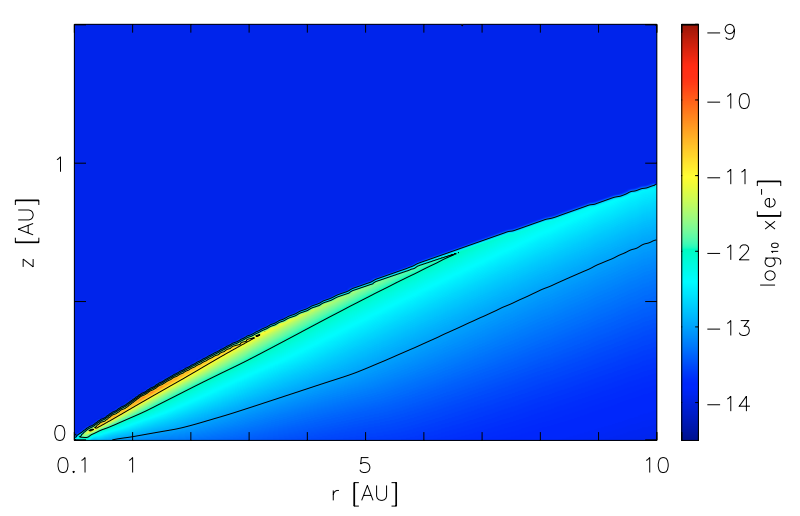

Fig. 21. This contour plot shows the electron distribution at $t=10^{5} \mathrm{yrs}$ for our $\alpha=10^{-2}, \dot{M}=10^{-7} M_{\odot} \mathrm{yr}^{-1}$ disk model by applying the Oppenheimer \& Dalgarno model (model1) with $x_{\mathrm{Mg}}=10^{-12}$ and modified rate coefficients $\tilde{\alpha}$ and $\tilde{\gamma}$. Compared with the values in Table $1, \tilde{\alpha}$ and $\tilde{\gamma}$ are increase by 10 and 200 , respectively. The contour lines refer to values $x\left[\mathrm{e}^{-}\right]$of $10^{-13}, 10^{-12}$, and $10^{-11}$.

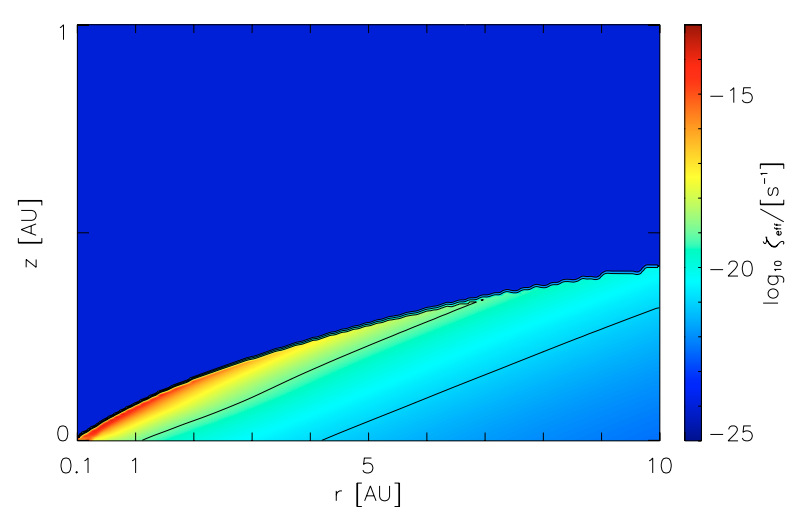

Fig. 22. The effective $X$-ray ionisation rate $\zeta_{\text {eff }}$ per hydrogen nucleus. The disk parameters are $\alpha=5 \times 10^{-3}$ and $\dot{M}=10^{-8} M_{\odot} \mathrm{yr}^{-1}$. The contour lines refer to values of $\zeta_{\mathrm{eff}}: 10^{-19}$ and $10^{-21} \mathrm{~s}^{-1}$.

and density of this disk model mean that the ionisation rate is now higher. The mass of the disk within the computational domain is now about $0.0049 M_{\odot}$ compared with $0.0087 M_{\odot}$ for the heavier disk model.

By applying model7 we calculated the ionisation fraction for $x_{\mathrm{gr}}=0$ and $x_{\mathrm{gr}}=10^{-12}$. The column densities of the active zone are shown in Fig. 23. We simply comment that a greater percentage of the disk is active when the surface density is reduced, but when grains are absent even a relatively large abundance of metals is unable to render the disk completely active. When grains are present, however, it remains the case that only about $1 \%$ of the matter beyond $R \simeq 1 \mathrm{AU}$ is magnetically active according to our adopted criterion.

\section{Summary}

We have presented calculations of the ionisation fraction in $\alpha$ disk models using a number of chemical reaction networks that have appeared in the literature. The primary aims are: to compare the predictions of these networks for the ionisation degree in identical disk models; to examine the role of gas-grain 


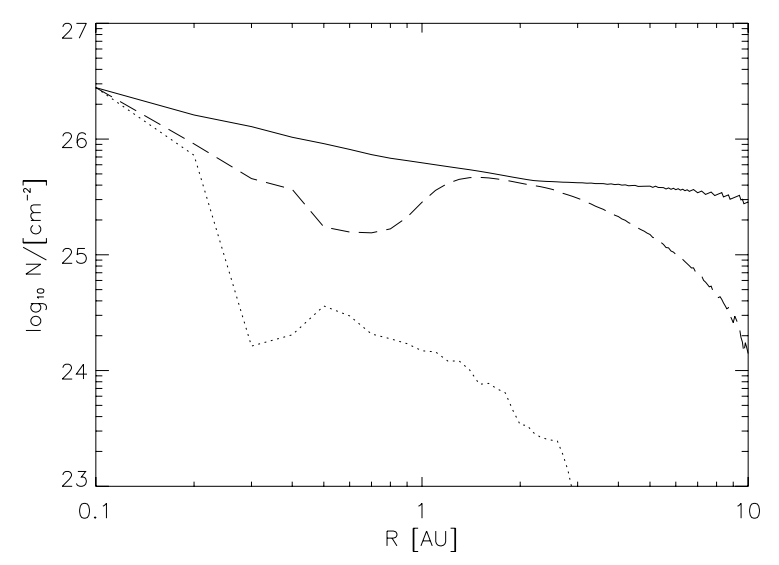

Fig. 23. model 7 - Column densities of the whole disk (solid line) and of the active zones (dashed and dotted lines) - referring to magnetic Reynolds numbers greater than 100 - for different values of $x_{\mathrm{gr}}$. While the dashed line refers to $x_{\mathrm{gr}}=0$ (gas-phase ch.), the dotted line refers to $x_{\mathrm{gr}}=10^{-12}$ (gas-grain ch.). Values of $x_{\mathrm{Fe}}=2.74 \times 10^{-9}$ and $x_{\mathrm{Mg}}=$ $1.09 \times 10^{-8}$ are used. The disk parameters are $\alpha=5 \times 10^{-3}$ and $\dot{M}=$ $10^{-8} M_{\odot} \mathrm{yr}^{-1}$.

chemistry in determining the ionisation fraction in protoplanetary disks; to examine the level of grain depletion required to converge toward the pure gas-phase chemistry. Our main conclusions are:

(1) Solving Eq. (1) to obtain the electron fraction, using the approximation that the neutral metal abundance is constant, leads to an overestimate of the size of the active zone when heavy metals are included in the gas.

(2) In agreement with previous work (Fromang et al. 2002), we find that the simple Oppenheimer \& Dalgarno (1974) reaction network predicts globally active disks when the elemental abundance of metals exceeds $10^{-11}$ and the disk parameters are $\dot{M}=10^{-7} M_{\odot} \mathrm{yr}^{-1}$ and $\alpha=10^{-2}$.

(3) A complex gas-phase chemistry drawn from the UMIST data base predicts larger dead zones than the simple Oppenheimer \& Dalgarno (1974) model. Whereas disks can be rendered fully active by the addition of gas-phase heavy metals to the simple model, the complex model predicts extensive dead zones even for $x_{\mathrm{Mg}}=10^{-8}$.

(4) A procedure for fitting the results of the complex gasphase model, using a modification of the Oppenheimer \& Dalgarno scheme, can be successful for specific models, but breaks down when the abundance of gas-phase metals is increased above $x_{\mathrm{Mg}}=10^{-12}$.

(5) We find that the addition of small grains with concentration $x_{\mathrm{gr}}=10^{-12}$ leads to a dramatic increase in the size of the dead zone for all gas-grain chemical models considered.

(6) In contrast to the gas-phase chemistry, there is very good agreement in the predicted size and structure of the active zone between the different gas-grain chemical networks. This is because the grains play a dominant role in all gasgrain schemes.

(7) A grain depletion factor of $\sim 10^{-4}$ is required to reproduce the metal-free gas phase chemistry.

(8) A grain depletion factor of $\simeq 10^{-8}$ is required to reproduce the gas phase chemistry including metals.
Taken at face value, conclusions (7) and (8) suggest that efficient growth and settling of small grains will be required if gas-phase chemical models are to be used to calculate the ionisation structure of protoplanetary disks. Furthermore, this requirement must also be met before a significant mass fraction of a protoplanetary disk can sustain MHD turbulence. In a recent study, Dullemond \& Dominik (2005) calculated the growth and settling of grains in laminar and turbulent disks, demonstrating that theory predicts the rapid removal of small, submicron sized grains. Depletion factors of $<10^{-6}$ were obtained within $\simeq 10^{4} \mathrm{yr}$, with the consequences for observation and theory being: the disk is rendered optically thin to the UV (which has potential implications for the chemistry and ionisation fraction); the $10 \mu \mathrm{m}$ silicate feature is reduced or removed from the spectral energy distribution; the mid-IR emission undergoes rapid decline. The latter two consequences are at odds with T Tauri disk observations (Dullemond \& Dominik 2005), which maintain submicron grain populations for longer than $10^{6}$ yr. A plausible scenario is that an initially quiescent disk undergoes grain growth and settling, leading to the development of MHD turbulence whose strength is modulated by the gas-phase electron fraction. Turbulent motions may contribute to the fragmentation of solids, as well as grain growth, so that a population of small grains is maintained in the disk. Feedback between grain size distribution and the strength of the turbulence may lead to a quasi-steady state in which the rate of growth and fragmentation of solids is controlled by the turbulence, whose strength is in turn controlled by the grain size distribution. Further work is clearly required to test the validity of such a scenario.

In this paper we have concentrated on calculating quasi steady-state chemical and ionisation profiles in simple disk models, ignoring potentially important effects such as turbulent mixing, diffusion, and a time dependent X-ray flux. We have already constructed models including some of these effects, based on the reaction networks that we have described in this paper. These more complex models are described in Ilgner \& Nelson (2005).

Acknowledgements. This research was supported by the European Community's Research Training Networks Programme under contract HPRN-CT-2002-00308, "PLANETS".

\section{References}

Sicilia-Aguilar, A., Hartmann, L., Briceño, C., Muzerolle, J., \& Calvet, N. 2004, AJ, 128, 805

Balbus, S., \& Hawley, J. 1991, ApJ, 376, 214

Balbus, S., \& Hawley, J. 1998, Rev. Mod. Phys., 70, 1

Blaes, O., \& Balbus, S. 1994, ApJ, 421, 163

Chiang, E., \& Goldreich, P. 1997, ApJ, 490, 368

Cravens, T., \& Dalgarno, A. 1978, ApJ, 219, 750

D’Alessio, P., Cantó, J., Calvet, N., \& Lizano, S. 1998, ApJ, 500, 411

Dalgarno, A., Oppenheimer, M., \& Berry, R. 1973, ApJ, 183, L21

Dullemond, C., \& Dominik, C. 2005, A\&A, 434, 971

Draine, B., \& Sutin, B. 1987, ApJ, 320, 803

Fleming, T., Stone, J., \& Hawley, J. 2000, ApJ, 530, 464

Fromang, S., Terquem, C., \& Balbus, S. 2002, MNRAS, 329, 18

Glassgold, A., Najita, J., \& Igea, J. 1997, ApJ, 480, 344 
Glassgold, A., Feigelson, E., \& Montmerle, T. 2000, Protostars and Planets IV, ed. V. Mannings, A. Boss, S. Russell, 429

Hasegawa, T., \& Herbst, E. 1993, MNRAS, 261, 83

Hasegawa, T., Herbst, E., \& Leung, C. 1992, ApJS, 82, 167

Hawley, J., \& Balbus, S. 1991, ApJ, 376, 223

Hawley, J., Gammie, C., \& Balbus, S. 1996, ApJ, 464, 690

Hayashi, C. 1981, Prog. Theor. Phys. Suppl., 70, 35

Hollenbach, D., \& Salpeter, E. 1970, J. Chem. Phys., 53, 79

Igea, J., \& Glassgold, A. 1999, ApJ, 518, 848

Ilgner, M., \& Nelson, R. 2005, A\&A, 445, 223

Ilgner, M., Henning, Th., Markwick, A., \& Millar, T. 2004, A\&A, 415, 643

Kokubo, E., \& Ida, S. 2000, Icarus, 143, 15

Le Teuff, Y., Millar, T., \& Markwick, A. 2000, A\&AS, 146, 157

Markwick, A., Ilgner, M., Millar, T., \& Henning, Th. 2002, A\&A, 385, 632

Matsumura, S., \& Pudritz, R. 2003, ApJ, 598, 645

Matsumura, S., \& Pudritz, R. 2005, ApJ, 618, L137

Najita, J., Bergin, T., \& Ullom, J. 2001, ApJ, 561, 880

Nelson, R. 2005, A\&A, in press

Nelson, R., \& Papaloizou, J. 2003, MNRAS, 339, 993
Nelson, R., \& Papaloizou, J. 2004, MNRAS, 350, 849

Nishi, R., Nakano, T., \& Umebayashi, T. 1991, ApJ, 368, 181

Oppenheimer, M., \& Dalgarno, A. 1974, ApJ, 192, 29

Papaloizou, J., \& Terquem, C. 1999, ApJ, 521, 823

Sandford, S., \& Allamandola, L. 1993, ApJ, 417, 815

Sano, T., \& Stone, J. 2002, ApJ, 577, 534

Sano, T., Miyama, S., Umebayashi, T., \& Nakano, T. 2000, ApJ, 543, 486

Semenov, D., Henning, Th., Helling, Ch., Ilgner, M., \& Sedlmayer, E. 2003, A\&A, 410, 611

Semenov, D., Wiebe, D., \& Henning, Th. 2004, A\&A, 417, 93

Shang, H., Glassgold, A., Shu, F., \& Lizano, S. 2002, ApJ, 564, 853

Thommes, E., Duncan, M., \& Levison, H. 2003, Icarus, 161, 431

Umebayashi, T., \& Nakano, T. 1990, MNRAS, 243, 103

Umebayashi, T., \& Nakano, T. 1980, Publ. Astron. Soc. Japan, 32, 405

Wetherill, G., \& Stewart, G. 1993, Icarus 106, 190

Willacy, K., \& Langer, W. 2000, ApJ, 544, 903

Willacy, K., Klahr, H., Millar, T., \& Henning, Th. 1998, A\&A, 338, 995

Winters, W., Balbus, S., \& Hawley, J. 2003, ApJ., 589, 543

Yamamoto, T., Nakagawa, N., \& Fukui, Y. 1983, A\&A, 122, 171 


\section{Online Material}




\section{Appendix A:}

\section{A.1. List of gaseous components and grain particles}

We considered the following elements: $\mathrm{H}, \mathrm{He}, \mathrm{C}, \mathrm{N}, \mathrm{O}, \mathrm{Mg}, \mathrm{Si}$, $\mathrm{S}, \mathrm{Fe}$.

\section{A.2. Kinetic model of the adsorbed components}

Collisions of gaseous components with grain particles may lead to adsorption of the gaseous components onto the surfaces of the grain particles. According to the basic assumption of kinetics, the adsorbed counterpart $\mathrm{gX}$ of the gaseous species $\mathrm{X}$ is considered as an individual component. In addition, the adsorbed species $\mathrm{gX}$ is characterized by the grain charge itself. Restricting to neutral and simply charged grains, the adsorbed species $\mathrm{X}(\mathrm{gr}), \mathrm{X}\left(\mathrm{gr}^{-}\right)$, and $\mathrm{X}\left(\mathrm{gr}^{+}\right)$have to be considered as individual components so far. However, it becomes important if processes between ionized gaseous species, $\mathrm{X}^{+}$, and grain particles are described by the kinetic model.

Processes onto grain surfaces which involve neutral and ionized gaseous species are listed in Table 3. Note, the notation, e.g., $\mathrm{X}\left(\mathrm{gr}^{-}\right)$refers to an adsorbed species of a neutral gaseous counterpart $\mathrm{X}$ onto a grain with simply negative charge. In addition be aware that not every reaction listed in Table 3 may be applied for each gaseous species; cf. for example $\mathrm{NH}_{4}^{+}$.

Three different processes are considered: i) adsorption processes - reactions 1 to 3 -; ii) desorption processes - reactions 6 to 8 ; and iii) collisional charging processes - reactions 4 and 5 .

Applying the mass action kinetic deterministic model of the reactions listed in Table A.3, one can derive a single ordinary differential equation for each component. The number of ordinary differential equations can be reduced - which becomes important for systems with a large number of gaseous components - if one considers the total contributions of the mantle components only. Taking

$\bar{k}_{1} \equiv k_{1}=k_{2}=k_{3}$

$\bar{k}_{2} \equiv k_{4}$

$\bar{k}_{3} \equiv k_{5}$

$\bar{k}_{4} \equiv k_{6}=k_{7}=k_{8}$

into account (cf. Sect. 3.2) one gets

$\frac{\mathrm{d}}{\mathrm{d} t} n[\mathrm{X}]=\bar{k}_{2} n\left[\mathrm{X}^{+}\right] n\left[\mathrm{gr}^{-}\right]+\bar{k}_{4} n[\mathrm{gX}]-\bar{k}_{1} n[\mathrm{X}] n_{\mathrm{gr}}$

$\frac{\mathrm{d}}{\mathrm{d} t} n\left[\mathrm{X}^{+}\right]=-\bar{k}_{2} n\left[\mathrm{X}^{+}\right] n\left[\mathrm{gr}^{-}\right]-\bar{k}_{3} n\left[\mathrm{X}^{+}\right] n[\mathrm{gr}]$

$\frac{\mathrm{d}}{\mathrm{d} t} n[\mathrm{gX}]=\bar{k}_{1} n[\mathrm{X}] n_{\mathrm{gr}}+\bar{k}_{3} n\left[\mathrm{X}^{+}\right] n[\mathrm{gr}]-\bar{k}_{4} n[\mathrm{gX}]$

$\frac{\mathrm{d}}{\mathrm{d} t} n\left[\mathrm{gr}^{-}\right]=-\bar{k}_{2} n\left[\mathrm{X}^{+}\right] n\left[\mathrm{gr}^{-}\right]$

$\frac{\mathrm{d}}{\mathrm{d} t} n[\mathrm{gr}]=\bar{k}_{2} n\left[\mathrm{X}^{+}\right] n\left[\mathrm{gr}^{-}\right]-\bar{k}_{3} n\left[\mathrm{X}^{+}\right] n[\mathrm{gr}]$

$\frac{\mathrm{d}}{\mathrm{d} t} n\left[\mathrm{gr}^{+}\right]=\bar{k}_{3} n\left[\mathrm{X}^{+}\right] n[\mathrm{gr}]$,

with $n[\mathrm{gX}]=n\left[\mathrm{X}\left(\mathrm{gr}^{-}\right)\right]+n[\mathrm{X}(\mathrm{gr})]+n\left[\mathrm{X}\left(\mathrm{gr}^{+}\right)\right]$and $n_{\mathrm{gr}}=$ $n\left[\mathrm{gr}^{-}\right]+n[\mathrm{gr}]+n\left[\mathrm{gr}^{+}\right]$. The quantity $n[\ldots]$ refers to the particle number density per $\mathrm{cm}^{3}$.
Table A.1. List of gaseous species including free electrons and grain particles with different electric excess charges.

\begin{tabular}{|c|c|c|c|c|}
\hline$\overline{\overline{\mathrm{H}^{+}}}$ & $\overline{\mathrm{HH}}$ & $\overline{\overline{\mathrm{H}_{2}}}$ & $\overline{\mathrm{H}_{2}^{+}}$ & $\overline{\overline{\mathrm{H}_{3}^{+}}}$ \\
\hline $\mathrm{He}$ & $\mathrm{He}^{+}$ & $\mathrm{C}^{2}$ & $\mathrm{C}^{+}$ & $\mathrm{CH}$ \\
\hline $\mathrm{CH}^{+}$ & $\mathrm{CH}_{2}$ & $\mathrm{CH}_{2}^{+}$ & $\mathrm{N}^{+}$ & $\mathrm{N}$ \\
\hline $\mathrm{CH}_{3}^{+}$ & $\mathrm{NH}^{+}$ & $\mathrm{CH}_{3}$ & $\mathrm{NH}$ & $\mathrm{CH}_{4}^{+}$ \\
\hline $\mathrm{NH}_{2}^{+}$ & $\mathrm{O}$ & $\mathrm{NH}_{2}$ & $\mathrm{O}^{+}$ & $\mathrm{CH}_{4}$ \\
\hline $\mathrm{OH}$ & $\mathrm{NH}_{3}$ & $\mathrm{NH}_{3}^{+}$ & $\mathrm{OH}^{+}$ & $\mathrm{CH}_{5}^{+}$ \\
\hline $\mathrm{H}_{2} \mathrm{O}$ & $\mathrm{NH}_{4}^{+}$ & $\mathrm{H}_{2} \mathrm{O}^{+}$ & $\mathrm{H}_{3} \mathrm{O}^{+}$ & $\mathrm{Mg}$ \\
\hline $\mathrm{C}_{2}$ & $\mathrm{C}_{2}^{+}$ & $\mathrm{Mg}^{+}$ & $\mathrm{C}_{2} \mathrm{H}^{+}$ & $\mathrm{C}_{2} \mathrm{H}$ \\
\hline $\mathrm{CN}$ & $\mathrm{C}_{2} \mathrm{H}_{2}$ & $\mathrm{CN}^{+}$ & $\mathrm{C}_{2} \mathrm{H}_{2}^{+}$ & $\mathrm{HCN}$ \\
\hline $\mathrm{C}_{2} \mathrm{H}_{3}^{+}$ & HNC & $\mathrm{C}_{2} \mathrm{H}_{3}$ & $\mathrm{HCN}^{+}$ & $\mathrm{H}_{2} \mathrm{NC}^{+}$ \\
\hline $\mathrm{Si}^{+}$ & $\mathrm{HCNH}^{+}$ & $\mathrm{CO}^{+}$ & $\mathrm{CO}$ & $\mathrm{Si}^{2}$ \\
\hline $\mathrm{N}_{2}^{+}$ & $\mathrm{N}_{2}$ & $\mathrm{C}_{2} \mathrm{H}_{4}^{+}$ & $\mathrm{SiH}^{+}$ & $\mathrm{HCO}^{+}$ \\
\hline $\mathrm{HCO}$ & $\mathrm{SiH}$ & $\mathrm{HN}_{2}^{+}$ & $\mathrm{NO}^{+}$ & $\mathrm{H}_{2} \mathrm{CO}^{+}$ \\
\hline $\mathrm{SiH}_{2}$ & NO & $\mathrm{SiH}_{2}^{+}$ & $\mathrm{H}_{2} \mathrm{CO}$ & $\mathrm{H}_{3} \mathrm{CO}^{+}$ \\
\hline $\mathrm{SiH}_{3}$ & $\mathrm{SiH}_{3}^{+}$ & $\mathrm{S}^{+}$ & $\mathrm{CH}_{3} \mathrm{OH}^{+}$ & $\mathrm{SiH}_{4}$ \\
\hline $\mathrm{CH}_{3} \mathrm{OH}$ & $\mathrm{O}_{2}^{+}$ & $S$ & $\mathrm{SiH}_{4}^{+}$ & $\mathrm{O}_{2}$ \\
\hline $\mathrm{HS}^{+}$ & $\mathrm{CH}_{3} \mathrm{OH}_{2}^{+}$ & HS & $\mathrm{SiH}_{5}^{+}$ & $\mathrm{H}_{2}^{2} \mathrm{~S}$ \\
\hline $\mathrm{H}_{2} \mathrm{~S}^{+}$ & $\mathrm{H}_{3} \mathrm{~S}^{+}$ & $\mathrm{C}_{3}$ & $\mathrm{C}_{3}{ }^{-}$ & $\mathrm{C}_{3} \mathrm{H}$ \\
\hline $\mathrm{C}_{3} \mathrm{H}^{+}$ & $\mathrm{C}_{3} \mathrm{H}_{2}$ & $\mathrm{C}_{3} \mathrm{H}_{2}^{+}$ & $\mathrm{C}_{2}^{3} \mathrm{~N}^{+}$ & $\mathrm{CNC}^{+}$ \\
\hline $\mathrm{C}_{3} \mathrm{H}_{3}$ & $\mathrm{C}_{3} \mathrm{H}_{3}^{+}$ & $\mathrm{C}_{3} \mathrm{H}_{4}$ & $\mathrm{SiC}^{+}$ & $\mathrm{C}_{3} \mathrm{H}_{4}^{+}$ \\
\hline $\mathrm{SiC}$ & $\mathrm{C}_{2} \mathrm{O}^{+}$ & $\mathrm{HCSi}^{4}$ & $\mathrm{HC}_{2} \mathrm{O}^{+}$ & $\mathrm{HCSi}^{+}$ \\
\hline $\mathrm{CH}_{3} \mathrm{CN}^{+}$ & $\mathrm{C}_{3} \mathrm{H}_{5}^{+}$ & $\mathrm{CH}_{3} \mathrm{CN}$ & $\mathrm{CH}_{2} \mathrm{CO}$ & $\mathrm{SiN}^{+}$ \\
\hline $\mathrm{SiN}$ & $\mathrm{CH}_{2} \mathrm{CO}^{+}$ & $\mathrm{H}_{4} \mathrm{C}_{2} \mathrm{~N}^{+}$ & $\mathrm{CH}_{3} \mathrm{CO}^{+}$ & HNSi \\
\hline $\mathrm{HNSi}^{+}$ & $\mathrm{CO}_{2}^{+}$ & $\mathrm{SiO}^{+}$ & $\mathrm{CS}$ & $\mathrm{SiO}$ \\
\hline $\mathrm{CO}_{2}$ & $\mathrm{CS}^{+}$ & $\mathrm{HCS}$ & $\mathrm{HCO}_{2}^{+}$ & $\mathrm{SiOH}^{+}$ \\
\hline $\mathrm{HCS}^{+}$ & $\mathrm{NS}^{+}$ & NS & $\mathrm{H}_{2} \mathrm{CS}^{2}$ & $\mathrm{H}_{2} \mathrm{CS}^{+}$ \\
\hline $\mathrm{H}_{3} \mathrm{CS}^{+}$ & $\mathrm{HNS}^{+}$ & $\mathrm{C}_{4}^{+}$ & $\mathrm{SO}^{+}$ & SO \\
\hline $\mathrm{C}_{4}$ & $\mathrm{HSO}^{+}$ & $\mathrm{C}_{4} \mathrm{H}^{+}$ & $\mathrm{C}_{4} \mathrm{H}$ & $\mathrm{C}_{3} \mathrm{~N}$ \\
\hline $\mathrm{C}_{4}^{4} \mathrm{H}_{2}^{+}$ & $\mathrm{C}_{3} \mathrm{~N}^{+}$ & $\mathrm{C}_{4}^{4} \mathrm{H}_{2}$ & $\mathrm{HC}_{3} \mathrm{~N}^{+}$ & $\mathrm{HC}_{3} \mathrm{~N}$ \\
\hline $\mathrm{C}_{3} \mathrm{O}^{+}$ & $\mathrm{C}_{3} \mathrm{O}$ & $\mathrm{H}_{2} \mathrm{C}_{3}^{2} \mathrm{~N}^{+}$ & $\mathrm{HC}_{3} \mathrm{O}^{+}$ & $\mathrm{C}_{3} \stackrel{\vec{H}}{2}_{2} \mathrm{O}^{+}$ \\
\hline $\mathrm{H}_{3} \mathrm{C}_{3} \mathrm{O}^{+}$ & $\mathrm{C}_{2} \mathrm{~S}^{+}$ & $\mathrm{C}_{2} \mathrm{~S}$ & $\mathrm{Fe}^{+}$ & $\mathrm{Fe}$ \\
\hline $\mathrm{HC}_{2} \mathrm{~S}^{+}$ & $\mathrm{SiS}$ & $\mathrm{OCS}^{+}$ & $\mathrm{SiO}_{2}$ & $\mathrm{SiS}^{+}$ \\
\hline $\mathrm{OCS}$ & $\mathrm{HSiS}^{+}$ & $\mathrm{HOCS}^{+}$ & $\mathrm{SO}_{2}^{+}$ & $\mathrm{SO}_{2}$ \\
\hline $\mathrm{S}_{2}$ & $\mathrm{HSO}_{2}^{+}$ & $\mathrm{H}_{4} \mathrm{C}_{4} \mathrm{~N}^{+}$ & $\mathrm{H}_{2} \mathrm{~S}_{2}^{+}$ & $\mathrm{C}_{3} \mathrm{~S}$ \\
\hline $\mathrm{C}_{3} \mathrm{~S}^{+}$ & $\mathrm{HC}_{3} \mathrm{~S}^{+}$ & $\mathrm{C}_{7}^{+}$ & $\mathrm{e}^{-}$ & $\mathrm{gr}$ \\
\hline $\mathrm{gr}^{2-}$ & $\mathrm{gr}^{-}$ & $\mathrm{gr}^{+}$ & $\mathrm{gr}^{2+}$ & \\
\hline
\end{tabular}


M. Ilgner and R. P. Nelson: Ionisation fraction in disks. I., Online Material p 3

Table A.2. Evaporation energies of the considered components adsorbed onto grain surfaces. The values are tabulated in Hasegawa \& Herbst (1993), if not referred to elsewhere. Values marked by $*$ and $\dagger$ are listed in Sandford \& Allamandola (1993) and Yamamoto et al. (1983), respectively.

\begin{tabular}{|c|c|c|c|c|c|}
\hline$\overline{\overline{\mathrm{H}}}$ & 350.0 & $\overline{\mathrm{C}_{2} \mathrm{H}_{3}}$ & $\bar{~} 1760.0$ & $\mathrm{CO}_{2}$ & $2860.0^{*}$ \\
\hline $\mathrm{H}_{2}$ & 450.0 & $\mathrm{H}_{2} \mathrm{CO}$ & 1760.0 & $\mathrm{C}_{4} \mathrm{H}_{2}$ & 2920.0 \\
\hline $\mathrm{NH}$ & 604.0 & $\mathrm{H}_{2} \mathrm{~S}$ & 1800.0 & $\mathrm{SiH}$ & 2940.0 \\
\hline $\mathrm{CH}$ & 654.0 & NS & 2000.0 & $\mathrm{HC}_{3} \mathrm{~N}$ & 2970.0 \\
\hline $\mathrm{N}_{2}$ & $710.0^{\dagger}$ & SO & 2000.0 & OCS & 3000.0 \\
\hline $\mathrm{O}$ & 800.0 & $\mathrm{~S}_{2}$ & 2000.0 & $\mathrm{C}_{3} \mathrm{~S}$ & 3000.0 \\
\hline $\mathrm{N}$ & 800.0 & CS & 2000.0 & $\mathrm{NH}_{3}$ & $3075.0^{*}$ \\
\hline $\mathrm{C}$ & 800.0 & HCS & 2000.0 & $\mathrm{SiH}_{2}$ & 3190.0 \\
\hline $\mathrm{NH}_{2}$ & 856.0 & $\mathrm{C}_{3}$ & 2010.0 & $\mathrm{SiH}_{3}$ & 3440.0 \\
\hline $\mathrm{CH}_{2}$ & 956.0 & $\mathrm{C}_{3} \mathrm{H}_{2}$ & 2110.0 & $\mathrm{SO}_{2}$ & $3460.0^{*}$ \\
\hline $\mathrm{CH}_{4}^{2}$ & $1080.0^{\dagger}$ & $\mathrm{C}_{3} \mathrm{H}_{3}$ & 2220.0 & $\mathrm{SiO}^{2}$ & 3500.0 \\
\hline$S$ & 1100.0 & $\mathrm{H}_{2} \mathrm{CS}$ & 2250.0 & $\mathrm{SiC}$ & 3500.0 \\
\hline $\mathrm{CH}_{3}$ & 1160.0 & $\mathrm{C}_{3} \mathrm{H}$ & 2270.0 & HCSi & 3500.0 \\
\hline $\mathrm{O}_{2}$ & 1210.0 & $\mathrm{CH}_{3} \mathrm{CN}$ & 2270.0 & $\mathrm{SiN}$ & 3500.0 \\
\hline $\mathrm{C}_{2}$ & 1210.0 & $\mathrm{C}_{2} \stackrel{3}{\mathrm{H}}_{2}$ & $2400.0^{\dagger}$ & $\mathrm{HNSi}$ & 3500.0 \\
\hline $\mathrm{CO}$ & 1210.0 & $\mathrm{C}_{4}$ & 2420.0 & $\mathrm{SiO}_{2}$ & 3500.0 \\
\hline NO & 1210.0 & $\mathrm{C}_{3} \mathrm{H}_{4}$ & 2470.0 & $\mathrm{SiH}_{4}$ & 3690.0 \\
\hline $\mathrm{OH}$ & 1260.0 & $\mathrm{C}_{2} \mathrm{~S}$ & 2500.0 & SiS & 3800.0 \\
\hline $\mathrm{C}_{2} \mathrm{H}$ & 1460.0 & $\mathrm{C}_{3} \mathrm{O}$ & 2520.0 & $\mathrm{HCN}$ & $4170.0^{\dagger}$ \\
\hline HS & 1500.0 & $\mathrm{CH}_{2} \mathrm{CO}$ & 2520.0 & $\mathrm{Fe}$ & 4200.0 \\
\hline $\mathrm{CN}$ & 1510.0 & $\mathrm{C}_{4} \mathrm{H}$ & 2670.0 & $\mathrm{CH}_{3} \mathrm{OH}$ & $4235.0^{*}$ \\
\hline $\mathrm{HCO}$ & 1510.0 & $\mathrm{Si}$ & 2700.0 & $\mathrm{H}_{2} \mathrm{O}$ & $4815.0^{*}$ \\
\hline HNC & 1510.0 & $\mathrm{C}_{3} \mathrm{~N}$ & 2720.0 & $\mathrm{Mg}$ & 5300.0 \\
\hline
\end{tabular}

Table A.3. Mantle chemistry

\begin{tabular}{llll}
\hline \hline $1 . \mathrm{X}+\mathrm{gr}^{-}$ & $\longrightarrow$ & $\mathrm{X}\left(\mathrm{gr}^{-}\right)$ \\
$2 . \mathrm{X}+\mathrm{gr}$ & $\longrightarrow$ & $\mathrm{X}\left(\mathrm{gr}^{2}\right)$ \\
$3 . \mathrm{X}+\mathrm{gr}^{+}$ & $\longrightarrow$ & $\mathrm{X}\left(\mathrm{gr}^{+}\right)$ \\
$4 . \mathrm{X}^{+}+\mathrm{gr}^{-}$ & $\longrightarrow$ & $\mathrm{X}$ \\
$5 . \mathrm{X}^{+}+\mathrm{gr}$ & $\longrightarrow$ & $\mathrm{X}\left(\mathrm{gr}^{+}\right)$ \\
$6 . \mathrm{X}\left(\mathrm{gr}^{-}\right)$ & $\longrightarrow$ & $\mathrm{X}$ \\
7. $\left(\mathrm{gr}^{+}\right.$ & $\longrightarrow$ & $\mathrm{X}$ \\
$8 . \mathrm{X}\left(\mathrm{gr}^{+}\right)$ & $\longrightarrow$ & $\mathrm{X}$ \\
\hline
\end{tabular}

